MATHEMATICS OF COMPUTATION

Volume 74, Number 250 , Pages $905-926$

S $0025-5718(04) 01721-1$

Article electronically published on October 29, 2004

\title{
COMPUTING ISOGENY COVARIANT DIFFERENTIAL MODULAR FORMS
}

\author{
CHRIS HURLBURT
}

\begin{abstract}
We present the computation modulo $p^{2}$ and explicit formulas for the unique isogeny covariant differential modular form of order one and weight $\chi-p-1,-p$ called $f_{\text {jet }}$, an isogeny covariant differential modular form of order two and weight $\chi_{-p^{2}-p,-1,-1}$ denoted by $f_{\text {jet }} h_{\text {jet }}$, and an isogeny covariant differential modular form $h_{\text {jet }}$ of order two and weight $\chi_{1-p^{2}, 0,-1}$.
\end{abstract}

\section{INTRODUCTION}

In this paper we introduce explicit formulas modulo $p^{2}$ for various differential modular forms discussed by Buium in [3, 2], 44. The central modular form discussed is the unique, up to multiplication by an element in $\mathbb{Z}_{p}^{*}$, isogeny covariant differential modular form of order one and weight $\chi_{-p-1,-1}$ called $f_{\text {jet }}, f_{p}^{1}$, and $f_{\text {jet }}^{1}$, respectively, in [3], [2], [4]. For the rest of this paper we mean "unique up to multiplication by an element in $\mathbb{Z}_{p}^{*}$ " when we say "unique", and we will refer to the unique isogeny covariant differential modular form of order one and weight $\chi_{-p-1,-1}$ by $f_{\text {jet }}$. This modular form has many interesting connections detailed in [1], 3], [2], and [4]. We compute $f_{\text {jet }}$ in a $p$-adic fashion following the construction of $f_{\text {jet }}$ detailed in [3] which allows us to compute modulo $p^{n}$ or specifically modulo $p^{2}$. Then we use the explicit formula from this computation to provide modulo $p^{2}$ formulas for order two differential modular forms. The specific order two isogeny covariant differential modular forms we describe are $f_{\text {jet }} h_{\text {jet }}$ from [3] also referred to as $k_{p}^{2}$ in [2] or $f_{\text {jet }}^{1,2}$ in [4] and $h_{\text {jet }}$ from [3] also referred to as $k_{p}^{2} / f_{p}^{1}$ in [2]. We note that modulo $p$ neither of these order two modular forms contain any second order terms.

The strategy is simple. We know that the isogeny covariant differential modular forms $f_{\text {jet }} h_{\text {jet }}$ and $h_{\text {jet }}$ of order two and weights $\chi_{-p^{2}-p,-1,-1}$ and $\chi_{1-p^{2}, 0,-1}$, respectively, are $f_{\text {jet }} h_{\text {jet }}=\phi\left(f_{\text {jet }}\right)$, where $\phi$ is the lifting of the Frobenius morphism, and outside the locus, where $f_{\text {jet }}$ modulo $p$ is zero $h_{\text {jet }}=\frac{\phi\left(f_{\text {jet }}\right)}{f_{\text {jet }}}[4]$. We should note that $h_{\text {jet }}$ is defined only outside this zero locus of $f_{\text {jet }}$ modulo $p$. In [5] we have the explicit computation of $\bar{f}_{\text {def }}$ (the $p$-derivation analog of the Kodaira-Spencer class) which is the reduction modulo $p$ of the unique isogeny covariant differential modular form of weight $\chi_{-p-1,-1}$. By uniqueness, $f_{\text {jet }} \equiv c \bar{f}_{\text {def }}$ modulo $p$ for some $c \in \mathbb{Z}_{p}^{*}$. Here we compute $f_{\text {jet }}$ directly allowing us to give a formula for the unique

Received by the editor January 14, 2004 and, in revised form, April 16, 2004.

2000 Mathematics Subject Classification. Primary 11F11; Secondary $12 \mathrm{H} 05$.

This research was supported in part by NSA grant MDA904-03-1-0031. 
isogeny covariant differential modular form modulo $p^{2}$ and not just modulo $p$. We also then are able to describe the order two terms that occur in $f_{\text {jet }} h_{\text {jet }}$ and $h_{\text {jet }}$ modulo $p^{2}$ but not modulo $p$.

For both context and notation we give the relevant definitions of differential modular forms. Let $p>3$ be a prime number. Let $M^{0}=\mathbb{Z}_{p}\left[a_{4}, a_{6}, \Delta^{-1}\right]^{\wedge}$, $M^{1}=\mathbb{Z}_{p}\left[a_{4}, a_{6}, \delta a_{4}, \delta a_{6}, \Delta^{-1}\right]^{\wedge}$, and $M^{2}=\mathbb{Z}_{p}\left[a_{4}, a_{6}, \delta a_{4}, \delta a_{6}, \delta^{2} a_{4}, \delta^{2} a_{6}, \Delta^{-1}\right]^{\wedge}$, where $\Delta=-2^{4}\left(4 a_{4}^{3}+27 a_{6}^{2}\right)$ and $\mathbb{Z}_{p}$ is the $p$-adic integer. We note that $a_{4}, a_{6}$, $\delta a_{4}, \delta a_{6}, \delta^{2} a_{4}, \delta^{2} a_{6}$ are variables over $\mathbb{Z}_{p}$ and that ${ }^{\wedge}$ represents the $p$-adic completion. Then the elements of $M^{1}$ are called $\delta$ modular forms of order one and elements of $M^{2}$ are called $\delta$ modular forms of order two as defined by Buium in [3].

Recall now that a $p$-derivation is a set theoretic map, $\delta: A \rightarrow B$, from a $\operatorname{ring} A$ to an $A$-algebra $B$ such that

$$
\begin{aligned}
\delta(x+y) & =\delta x+\delta y+C_{p}(x, y), \\
\delta(x y) & =y^{p} \delta x+x^{p} \delta y+p \delta x \delta y
\end{aligned}
$$

for all $x, y \in A$, where $C_{p}(X, Y)=\frac{X^{p}+Y^{p}-(X+Y)^{p}}{p}$. In Section 2 we will expand these axioms into a more complete list of properties of $p$-derivations. For now, if $A$ is a complete discrete valuation ring $R$, where $R$ has maximal ideal generated by $p$ and an algebraically closed residue field $k$, and if $\phi$ is the unique lifting of the Frobenius morphism to $A$, then the $p$-derivation given by $\delta(x)=\left(\phi(x)-x^{p}\right) / p$ is unique on $R$.

Now we use the $R$ and $\delta$ from our example and set

$$
M(R)=\left\{(a, b) \in R^{2} \mid 4 a^{3}+27 b^{2} \in R^{*}\right\} .
$$

Then the set $M(R)$ is in one-to-one correspondence with the set of pairs consisting of an elliptic curve over $R$ and an invertible 1-form; namely, each $\left(\bar{a}_{4}, \bar{a}_{6}\right) \in M(R)$ corresponds to $(E, d x / 2 y)$, where $E$ is the projective closure of the affine plane curved $y^{2}=x^{3}+\bar{a}_{4} x+\bar{a}_{6}$. For any $f \in M^{1}$, if we substitute $a, b, \delta a, \delta b$ in for $a_{4}, a_{6}, \delta a_{4}, \delta a_{6}$, then $f$ defines a map (still denoted by $f$ ) from $M(R)$ to $R$. This element in $M^{1}$ is in fact uniquely determined by the map from $M(R)$ to $R$. Similar statements are true for $f \in M^{2}$.

We define a $\delta$-character of order $\leq 1$ to be a group homomorphism $\chi: R^{*} \rightarrow R^{*}$ of the form $\chi=\chi_{m, n}$, where

$$
\chi_{m, n}(\lambda)=\lambda^{m}\left(\frac{\phi(\lambda)}{\lambda^{p}}\right)^{n} .
$$

Then a $\delta$-modular function of order one has weight $\chi$ if for any $\lambda \in R^{*}$

$$
f\left(\lambda^{4} a, \lambda^{6} b\right)=\chi(\lambda) f(a, b)
$$

for all $(a, b) \in M(R)$. We can easily extend the definition of $\delta$-characters to higher orders. Namely, a $\delta$-character of order $\leq 2$ is a group homomorphism $\chi: R^{*} \rightarrow R^{*}$ of the form $\chi=\chi_{m, n, r}$ where

$$
\chi_{m, n, r}(\lambda)=\lambda^{m}\left(\frac{\phi(\lambda)}{\lambda^{p}}\right)^{n}\left(\frac{\phi^{2}(\lambda)}{\lambda^{p^{2}}}\right)^{r} .
$$

The criterion for a $\delta$-modular function of order two to have weight $\chi$ is exactly the same as the criterion for a $\delta$-modular function of order one to have weight $\chi$. A $\delta$-modular form is a $\delta$-modular function with a weight. 
A $\delta$-modular form is isogeny covariant if for any two pairs $(a, b)$ and $(\tilde{a}, \tilde{b})$ with an etale isogeny of degree $N$ between the corresponding elliptic curves that pulls back $\frac{d x}{y}$ to $\frac{d x}{y}$

$$
f(a, b)=N^{-k / 2} f(\tilde{a}, \tilde{b}),
$$

where $k$ is a constant that depends solely on the weight. Note that for $\chi=\chi_{m, n}$ the constant is $k=m+n(1-p)$ and for $\chi=\chi_{m, n, r}$ the constant is $k=m+$ $n(1-p)+r\left(1-p^{2}\right)$.

Theorem 1.1. The isogeny covariant differential modular form of order one and weight $\chi_{-p-1,-1}$ modulo $p^{2}$ is

$$
f_{\text {jet }}=\left[\frac{-72 a_{6}^{p} \delta a_{4}+48 a_{4}^{p} \delta a_{6}}{\Delta^{p}}\right] \gamma_{2 p, p}+h+p H,
$$

where $\gamma_{2 p, p}$ and $h$ are polynomials in $M_{1}^{0}:=M^{0} \otimes \mathbb{Z}_{p} /\left(p^{2}\right), H$ is a polynomial in $M_{0}^{1}:=M^{1} \otimes \mathbb{Z}_{p} /(p)$, and $H$ is a nonhomogeneous quadratic in $\delta a_{4}$ and $\delta a_{6}$.

Explicit formulas for $h$ and $H$ are given in Theorem 6.11 and an explicit formula for $\gamma_{2 p, p}$ is given in Proposition 6.2.

Theorem 1.2. The isogeny covariant differential modular form $f_{\text {jet }} h_{\text {jet }}$ of order two and weight $\chi_{-p^{2}-p,-1,-1}$ modulo $p^{2}$ is

$$
\begin{aligned}
f_{\text {jet }} h_{\text {jet }}= & {\left[\frac{-72 a_{6}^{p^{2}}\left(\delta a_{4}\right)^{p}+48 a_{4}^{p^{2}}\left(\delta a_{6}\right)^{p}}{\Delta^{p^{2}}}\right] \gamma_{2 p, p}\left(a_{4}^{p}, a_{6}^{p}\right)+h^{*} } \\
& +p\left[\frac{-72 a_{6}^{p^{2}} \delta^{2} a_{4}+48 a_{4}^{p^{2}} \delta^{2} a_{6}}{\Delta^{p^{2}}}\right] \gamma_{2 p, p}\left(a_{4}^{p}, a_{6}^{p}\right)+p J,
\end{aligned}
$$

where $h^{*}$ is a polynomial in $M_{1}^{1}:=M^{1} \otimes \mathbb{Z}_{p} /\left(p^{2}\right)$ and $J$ is a polynomial in $M_{0}^{1}$.

Corollary 1.3. The isogeny covariant differential modular form $h_{\text {jet }}$ of order two and weight $\chi_{1-p^{2}, 0,-1}$ modulo $p^{2}$ is

$$
\begin{aligned}
& h_{\text {jet }}= \frac{\left[-72 a_{6}^{p^{2}}\left(\delta a_{4}\right)^{p}+48 a_{4}^{p^{2}}\left(\delta a_{6}\right)^{p}\right] \gamma_{2 p, p}\left(a_{4}^{p}, a_{6}^{p}\right)+\Delta^{p^{2}} h^{*}}{\Delta^{p^{2}-p}\left(\left[-72 a_{6}^{p} \delta a_{4}+48 a_{4}^{p} \delta a_{6}\right] \gamma_{2 p, p}+\Delta^{p} h\right)} \\
&+p\left(\frac{-\left(\left[-72 a_{6}^{p^{2}}\left(\delta a_{4}\right)^{p}+48 a_{4}^{p^{2}}\left(\delta a_{6}\right)^{p}\right] \gamma_{2 p, p}\left(a_{4}^{p}, a_{6}^{p}\right)+\Delta^{p^{2}} h^{*}\right) H}{\Delta^{p^{2}-2 p}\left(\left[-72 a_{6}^{p} \delta a_{4}+48 a_{4}^{p} \delta a_{6}\right] \gamma_{2 p, p}+\Delta^{p} h\right)^{2}}\right. \\
&\left.+\frac{\left[72 a_{6}^{p^{2}} \delta^{2} a_{4}+48 a_{4}^{p^{2}} \delta^{2} a_{6}\right] \gamma_{2 p, p}\left(a_{4}^{p}, a_{6}^{p}\right)+\Delta^{p^{2}} J}{\Delta^{p^{2}-p}\left(\left[-72 a_{6}^{p} \delta a_{4}+48 a_{4}^{p} \delta a_{6}\right] \gamma_{2 p, p}+\Delta^{p} h\right)}\right),
\end{aligned}
$$

where $h, h^{*}, H$, and $J$ are the same as in Theorems 1.1 and 1.2.

What follows is preliminary information to the calculation of $f_{\text {jet }}$. Let $E$ be the elliptic curve in Weierstrass form over $M^{0}$ defined by the homogeneous equation

$$
f(X, Y, W)=W Y^{2}-X^{3}-a_{4} X W^{2}-a_{6} W^{3} .
$$

Let $U$ and $V$ be the affine open subsets of $E$ given by the equations $f(x, y, 1)$ and $f(z, 1, w)$, respectively. So

$$
\begin{aligned}
& U=\operatorname{Spec} M^{0}[X, Y] /(f(X, Y, 1))=\operatorname{Spec} M^{0}[x, y], \\
& V=\operatorname{Spec} M^{0}[Z, W] /(f(Z, 1, W))=\operatorname{Spec} M^{0}[z, w],
\end{aligned}
$$


and on $U \cap V$

$$
\begin{aligned}
z & =-x / y, \\
w & =-1 / y,
\end{aligned}
$$

whence $E=U \cup V$. Next we define the first jets of $U$ and $V$ to be the sets

$$
\begin{aligned}
U^{1} & =\operatorname{Spec} M^{1}[X, Y, \delta X, \delta Y] /(f(X, Y, 1), \delta f(X, Y, 1))=\operatorname{Spec} M^{1}[x, y, \delta x, \delta y], \\
V^{1} & =\operatorname{Spec} M^{1}[Z, W, \delta Z, \delta W] /(f(Z, 1, W), \delta f(Z, 1, W))=\operatorname{Spec} M^{1}[z, w, \delta z, \delta w] .
\end{aligned}
$$

Then $E^{1}$, the first jet space of $E$, is the gluing of $U^{1}$ and $V^{1}$ by the maps

$$
\begin{aligned}
z & =-x / y, \\
w & =-1 / y, \\
\delta z & =\frac{x^{p} \delta y-y^{p} \delta x}{y^{p}\left(y^{p}+p \delta y\right)}, \\
\delta w & =\frac{\delta y}{y^{p}\left(y^{p}+p \delta y\right)} .
\end{aligned}
$$

We can extend the group law on $E$ to a group law on $E^{1}$. The group law arises naturally by construction from the group law on $E$ just as $E^{1}$ arises naturally by construction from $E$. This will be detailed explicitly in Section 3

From now on we will also use the following notation. First by $M_{n}^{i}$ we mean $M^{i} \otimes \mathbb{Z}_{p} /\left(p^{n+1}\right)$. For example $M_{0}^{1}=\mathbb{F}_{p}\left[a_{4}, a_{6}, \delta a_{4}, \delta a_{6}, \Delta^{-1}\right]$, where $\mathbb{F}_{p}$ is the finite field of $p$ elements. Second by $E_{m}^{1}$ we mean $E^{i} \otimes M_{m}^{1}$, and by $E_{m}=E_{m}^{0}$ we mean $E \otimes M_{m}^{0}$. Also we will use $\delta\left(a_{4}\right)$ interchangeably for $\delta a_{4}, \delta\left(a_{6}\right)$ interchangeably for $\delta a_{6}$, etc.

To compute $f_{\text {jet }}$, the isogeny covariant $\delta$ modular form of weight $\chi_{-p-1,-1}$, we work from [2, Construction 4.1]. The same construction is also described in [4 and 3. First we find two sections $s_{U}$ and $s_{V}$ of the morphisms $U^{1} \rightarrow U \otimes M^{1}$ and $V^{1} \rightarrow V \otimes M^{1}$, respectively, such that $s_{U}$ defines a morphism from $U \otimes M^{1}$ to $U^{1}$ and $s_{V}$ defines a morphism from $V \otimes M^{1}$ to $V^{1}$. Then the difference of the sections under the group law induces a morphism $s_{U}-s_{V}: U \cap V \otimes M^{1} \rightarrow E^{1}$. Let $\zeta$ be the $\delta z$ coordinate in the difference $s_{U}-s_{V}$. By the $\delta z$ coordinate, we mean the image of $\delta z \in U^{1} \cap V^{1}$ under the ring homomorphism induced by the morphism $s_{U}-s_{V}: U \cap V \otimes M^{1} \rightarrow E^{1}$. Let $\log _{\mathcal{F}_{1}^{\phi^{1}}}(\xi)$ be the formal logarithm of the Frobenius twist of the formal group of the elliptic curve, namely

$$
\log _{\mathcal{F}_{1}^{\phi^{1}}}(\xi)=\xi+\frac{p \phi\left(c_{1}\right)}{2} \xi^{2}+\frac{p^{2} \phi\left(c_{2}\right)}{3} \xi^{3}+\cdots,
$$

where the $c_{i}$ are the coefficients of the power series expansion of the invariant differential [3]. Then $\log _{\mathcal{F}_{1}^{\phi^{1}}}(\zeta)$ is a cohomology class in $H^{1}\left(E \otimes M^{1}, \mathcal{O}\right) \simeq H^{1}(E, \mathcal{O}) \otimes$ $M^{1}$, and this resulting class has a representative of the form $\sum a_{n} y^{n}+x \sum b_{n} y^{n}+$ $x^{2} \sum e_{n} y^{n}$. The modular form $f_{\text {jet }}$ is the coefficient $e_{-1}$ of $x^{2} / y$ in this representative which is the residue of the cohomology class, namely the image of the cohomology class under the Serre duality pairing.

We will actually work modulo $p^{2}$ which means that our end result will be $f_{\text {jet }}$ modulo $p^{2}$. In fact $f_{\text {jet }} \in M^{1}$ is a restricted power series whose coefficients expand exponentially in the number of terms in each coefficient of a power of $p$. Therefore, the formulas necessary to express $f_{\text {jet }}$ modulo $p^{n}$ for $n>2$ are prohibitive in length. At this point we note that the formal logarithm of the Frobenius twist of the formal 
group of the elliptic curve modulo $p^{2}$ is in fact the identity. This certainly simplifies one step of the computation modulo $p^{2}$; however, for $n>4$ this formal logarithm is no longer trivial modulo $p^{n}$, meaning this step is not trivial for large $n$. As a preliminary step to computing $f_{\text {jet }}$ we detail some computation guidelines for $p$-derivations and the group law for $E^{1}$ modulo $p^{2}$.

\section{Properties of $p$-Derivations}

Recall that a $p$-derivation is a set theoretic map, $\delta: A \rightarrow B$, from a ring $A$ to an $A$-algebra $B$ with $\delta(1)=0$ such that

$$
\delta(x+y)=\delta x+\delta y+C_{p}(x, y), \quad \delta(x y)=y^{p} \delta x+x^{p} \delta y+p \delta x \delta y
$$

for all $x, y \in A$, where $C_{p}(X, Y)=\frac{X^{p}+Y^{p}-(X+Y)^{p}}{p}$. In the case when $A=B=R$, where $R$ is a complete discrete valuation ring with maximal ideal generated by $p$ and has an algebraically closed residue field, there is a unique $p$-derivation given by $\delta(x)=\left(\phi(x)-x^{p}\right) / p$, where $\phi$ is the unique lifting of the Frobenius morphism to $R$.

This definition implies that if $\varphi: A \rightarrow B$ is the ring homomorphism associated to $B$ being an $A$-algebra, then

$$
(\varphi, \delta): A \rightarrow W_{2}(B)
$$

is a ring homomorphism, where $W_{2}(B)$ is the ring of Witt vectors of length two on $B$. With $(\varphi, \delta)$ as above, $\phi: A \rightarrow B$ defined by $\phi(x)=\varphi(x)^{p}+p \delta(x)$ is a ring homomorphism. In case $B=A$, this is a lifting of the Frobenius endomorphism $F(x)=x^{p}$ of $A / p A$.

While no further axioms for $p$-derivations beyond those in the definition are necessary for computation, the following $p$-derivation rules are very convenient for computation. Before introducing these rules we must define an extension of $C_{p}(X, Y)$.

Definition 2.1. For any $\sum q$, let

$$
C_{p}^{\mathrm{ext}}\left(\sum q\right)=\frac{\sum q^{p}-\left(\sum q\right)^{p}}{p}
$$

Note that $C_{p}^{\text {ext }}(X+Y)=\frac{X^{p}+Y^{p}-(X+Y)^{p}}{p}=C_{p}(X, Y)$; thus, this is a very natural definition.

Lemma 2.2. Let $\delta: A \rightarrow B$ be a $p$-derivation, let $g=\sum q, x, y \in A$, and let $n>0$ be an integer. Then the following are true.

(1) $\delta\left(\sum q\right)=\sum \delta q+C_{p}^{\operatorname{ext}}\left(\sum q\right)$.

(2) $\delta(-1)=0$.

(3) $\delta(-x)=-\delta x$.

(4) $\delta\left(x^{n}\right)=\sum_{k=1}^{n}\left(\begin{array}{l}n \\ k\end{array}\right) p^{k-1} x^{(n-k) p}(\delta x)^{k}=\frac{-x^{n p}+\left(x^{p}+p \delta x\right)^{n}}{p}$.

(5) $\delta\left(\frac{1}{x}\right)=\frac{-\delta x}{x^{p}\left(x^{p}+p \delta x\right)}$.

(6) $\delta\left(\frac{y}{x}\right)=\frac{x^{p} \delta y-y^{p} \delta x}{x^{p}\left(x^{p}+p \delta x\right)}$. 


\section{The Group LAW FOR The FIRST $p$-Jet SPACE OF $E$}

We now want to make the group law on $E^{1}$ explicit. This is necessary since the main result requires us to subtract two sections using the group law. The group law on the first $p$-jet is induced by the group law on the elliptic curve $E$, so we start by giving the group law on $E$. Let $\rho$ and $\psi$ be the equations that define the group law on $E$. So if $\left(z_{1}, w_{1}\right) \oplus\left(z_{2}, w_{2}\right)=\left(z_{3}, w_{3}\right)$, then

$$
\begin{aligned}
z_{3} & =\rho\left(z_{1}, w_{1}, z_{2}, w_{2}\right), \\
w_{3} & =\psi\left(z_{1}, w_{1}, z_{2}, w_{2}\right) .
\end{aligned}
$$

Then the group law on $E^{1}$ is an extension of the group law on $E$ such that if $\left(z_{1}, w_{1}, \delta z_{1}, \delta w_{1}\right) \oplus\left(z_{2}, w_{2}, \delta z_{2}, \delta w_{2}\right)=\left(z_{3}, w_{3}, \delta z_{3}, \delta w_{3}\right)$, then

$$
\begin{aligned}
z_{3} & =\rho\left(z_{1}, w_{1}, z_{2}, w_{2}\right), \\
w_{3} & =\psi\left(z_{1}, w_{1}, z_{2}, w_{2}\right), \\
\delta z_{3} & =\delta\left(\rho\left(z_{1}, w_{1}, z_{2}, w_{2}\right)\right), \\
\delta w_{3} & =\delta\left(\psi\left(z_{1}, w_{1}, z_{2}, w_{2}\right)\right) .
\end{aligned}
$$

To find appropriate $\rho$ and $\psi$, we must consider actual formulas for the group law. On the elliptic curve $E$, the group law can be explicitly formulated using the chord-tangent approach. In this approach we consider that every line intersects the elliptic curve at exactly three points counting multiplicity. We choose a specific point, $O$, to be the origin; in this case the point we choose to be the origin is the point at infinity, $(0,1,0)$. We then define the inverse of a point $P$ to be the third point on the line that intersects $P$ and the origin. We denote this point by $-P$. So if we want to add $P \oplus Q$, we take the line through $P$ and $Q$ and let $R$ be the third point on the line. Then we define $P \oplus Q=-R$. This definition arises naturally from the theory of Weil divisors. We refer to the case when $P=Q$ as the tangent case and $P \neq Q$ as the tangent case. From now on we will focus on the chord case of the chord-tangent approach since that is the most general case and the case used when computing the group law for a $p$-jet space.

We use the standard procedure for finding explicit formulas for group law in the $z$ and $w$ coordinates. In these coordinates our origin is $(0,0)$. To start with, we recall data on $V$; namely, that $f(z, 1, w)=w-z^{3}-a_{4} z w^{2}-a_{6} w^{3}$ is the curve we will be using and that any line through $P=\left(z_{0}, w_{0}\right)$ and the origin of $(0,0)$ will intersect $f(z, 1, w)$ at the third point $\left(-z_{0},-w_{0}\right)$. Whence $-P=\left(-z_{0},-w_{0}\right)$.

Now consider two points $P_{1}$ and $P_{2}$ denoted by $\left(z_{i}, w_{i}\right)$ for $i=1,2$, respectively. If we assume that $z_{1} \neq z_{2}$, the line connecting these two points is

$$
w=\frac{w_{2}-w_{1}}{z_{2}-z_{1}}\left(z-z_{1}\right)+w_{1}
$$

To find the sum $P_{1} \oplus P_{2}$, we must find the three points counting multiplicity of the intersection of this line with the curve $f(z, 1, w)$. If we substitute the line into the curve $f(z, 1, w)$ we get a cubic equation in terms of $z$. Finding these three points becomes a matter of finding the roots of the resulting cubic equation. On the other hand, we already know two of the roots, namely $z=z_{1}$ and $z=z_{2}$. The third root 
is

$$
z=\frac{-2 w_{2} z_{1}-w_{1} z_{1}+2 w_{1} z_{2}-3 a_{6} w_{2} w_{1}^{2} z_{2}-a_{4} w_{1}^{2} z_{2}^{2}+w_{2} z_{2}+3 w_{2}^{2} z_{1} a_{6} w_{1}+w_{2}^{2} z_{1}^{2} a_{4}}{3 a_{6} w_{2} w_{1}\left(w_{2}-w_{1}\right)+3 z_{2} z_{1}\left(z_{2}-z_{1}\right)+a_{4}\left(w_{2}^{2} z_{1}-w_{1}^{2} z_{2}\right)+w_{1}-w_{2}+2 a_{4} w_{2} w_{1}\left(z_{2}-z_{1}\right)}
$$

So for $z_{1} \neq z_{2}$, the $P_{3}=P_{1} \oplus P_{2}$ has coordinates

$$
\begin{aligned}
& z_{3}=-\frac{-2 w_{2} z_{1}-w_{1} z_{1}+2 w_{1} z_{2}-3 a_{6} w_{2} w_{1}^{2} z_{2}-a_{4} w_{1}^{2} z_{2}^{2}+w_{2} z_{2}+3 w_{2}^{2} z_{1} a_{6} w_{1}+w_{2}^{2} z_{1}^{2} a_{4}}{3 a_{6} w_{2} w_{1}\left(w_{2}-w_{1}\right)+3 z_{2} z_{1}\left(z_{2}-z_{1}\right)+a_{4}\left(w_{2}^{2} z_{1}-w_{1}^{2} z_{2}\right)+w_{1}-w_{2}+2 a_{4} w_{2} w_{1}\left(z_{2}-z_{1}\right)}, \\
& w_{3}=-\frac{3 w_{2} z_{2} z_{1}^{2}+z_{1} w_{2}^{2} w_{1} a_{4}-3 z_{1} w_{1} z_{2}^{2}+w_{1}^{2}-w_{2} w_{1}^{2} z_{2} a_{4}-w_{2}^{2}}{3 a_{6} w_{2} w_{1}\left(w_{2}-w_{1}\right)+3 z_{2} z_{1}\left(z_{2}-z_{1}\right)+a_{4}\left(w_{2}^{2} z_{1}-w_{1}^{2} z_{2}\right)+w_{1}-w_{2}+2 a_{4} w_{2} w_{1}\left(z_{2}-z_{1}\right)} .
\end{aligned}
$$

From this information, if we want the formulation of group law on $E^{1}$ we must simply take the $p$-derivation of these equations. The resulting group law for $P_{i}=$ $\left(z_{i}, w_{i}, \delta z_{i}, \delta w_{i}\right) \in V^{1}$ is

$$
\begin{aligned}
z_{3} & =-\frac{\alpha}{\mu}, \\
w_{3} & =-\frac{\beta}{\mu}, \\
\delta z_{3} & =-\frac{\mu^{p} \delta \alpha-\alpha^{p} \delta \mu}{\mu^{p}\left(\mu^{p}+p \delta \mu\right)}, \\
\delta w_{3} & =-\frac{\mu^{p} \delta \beta-\beta^{p} \delta \mu}{\mu^{p}\left(\mu^{p}+p \delta \mu\right)},
\end{aligned}
$$

where $P_{3}=P_{1} \oplus P_{2}$,

$$
\begin{aligned}
\alpha= & -2 w_{2} z_{1}-w_{1} z_{1}+2 w_{1} z_{2}-3 a_{6} w_{2} w_{1}^{2} z_{2} \\
& -a_{4} w_{1}^{2} z_{2}^{2}+w_{2} z_{2}+3 w_{2}^{2} z_{1} a_{6} w_{1}+w_{2}^{2} z_{1}^{2} a_{4}, \\
\beta= & 3 w_{2} z_{2} z_{1}^{2}+z_{1} w_{2}^{2} w_{1} a_{4}-3 z_{1} w_{1} z_{2}^{2}+w_{1}^{2}-w_{2} w_{1}^{2} z_{2} a_{4}-w_{2}^{2}, \\
\mu= & 3 a_{6} w_{2} w_{1}\left(w_{2}-w_{1}\right)+3 z_{2} z_{1}\left(z_{2}-z_{1}\right) \\
& +a_{4}\left(w_{2}^{2} z_{1}-w_{1}^{2} z_{2}\right)+w_{1}-w_{2}+2 a_{4} w_{2} w_{1}\left(z_{2}-z_{1}\right),
\end{aligned}
$$

and $\delta \alpha, \delta \beta, \delta \mu$ are the respective $p$-derivatives which are not included here because of their lengthy nature. On the other hand, this group law also describes the group law on $E_{m}^{1}$. For example, if $m=1$, then we consider this same group law modulo $p^{2}$. This does shorten the expressions of $\delta \alpha, \delta \beta$, and $\delta \mu$ for $m \leq 5$ to lengths that are possible to work with in computer algebra systems.

Besides shortening the expressions for $\alpha, \beta$, and $\mu$ one other advantage of explicitly detailing the group law on $E_{1}^{1}$ rather than $E^{1}$ is that we may write $\delta z_{3}$ and $\delta w_{3}$ in terms of polynomials in $\delta \alpha, \delta \beta$, and $\delta \mu$ by using their series expansions. Hence 
we have the following description for the group law on $E_{1}^{1}$ :

$$
\begin{aligned}
z_{3} & =-\frac{\alpha}{\mu}, \\
w_{3} & =-\frac{\beta}{\mu} \\
\delta z_{3} & =\frac{1}{\mu^{3 p}}\left(-\mu^{p} \delta \alpha+\alpha^{p} \delta \mu\right)\left(\mu^{p}-p \delta \mu\right), \\
\delta w_{3} & =\frac{1}{\mu^{3 p}}\left(-\mu^{p} \delta \beta+\beta^{p} \delta \mu\right)\left(\mu^{p}-p \delta \mu\right),
\end{aligned}
$$

where $\delta \alpha, \delta \beta$, and $\delta \mu$ are now expressions modulo $p^{2}$.

4. The SECTION ON $U$ THAT DEFINES A MAP FROM $U \otimes M^{1}$ TO $U^{1}$ AND THE SECTION ON $V$ THAT DEFINES A MAP FROM $V \otimes M^{1}$ TO $V^{1}$

We in fact want a specific map from $U$ to $U^{1}$; namely, the morphism which takes $\delta x$ and $\delta y$ to elements such that $\delta f(x, y, 1)$ is mapped to 0 . To do this we use a variant of Hensel's Lemma involving two variables which will be illuminated as we go along. To find the appropriate $\delta x$ and $\delta y$, we first consider the explicit expression of $\delta f(x, y, 1)$,

$$
\begin{aligned}
-p^{2} \delta x^{3} & +\left(-3 x^{p} \delta x^{2}-\delta a_{4} \delta x+\delta y^{2}\right) p \\
& +\left(-3 x^{2 p}-a_{4}^{p}\right) \delta x+2 y^{p} \delta y-\delta a_{4} x^{p}-\delta a_{6}+C_{p}^{\mathrm{ext}}\left(y^{2}-x^{3}-a_{4} x-a_{6}\right),
\end{aligned}
$$

and from this polynomial define $P_{U, 0}=-\delta a_{4} x^{p}-\delta a_{6}+C_{p}^{\operatorname{ext}}\left(y^{2}-x^{3}-a_{4} x-a_{6}\right)$. From now on for convenience of notation we will denote $f(x, y, 1)$ simply by $f$. We will also denote $\frac{\partial f}{\partial x}$ and $\frac{\partial f}{\partial y}$ by $f_{x}$ and $f_{y}$, respectively.

Now we let $A$ and $B$ be polynomials in $M^{0}[x, y]$ such that $A f_{x}+B f_{y}=1$. Specifically

$$
\begin{aligned}
& A=\frac{2^{4}\left(4 a_{4}^{2}+6 x^{2} a_{4}-9 x a_{6}\right)}{\Delta}, \\
& B=\frac{2^{3}(9 y)\left(2 x a_{4}-3 a_{6}\right)}{\Delta} .
\end{aligned}
$$

By simple arithmetic, $A^{p} f_{x}^{p}+B^{p} f_{y}^{p}=1+A^{p} f_{x}^{p}+B^{p} f_{y}^{p}-\left(A f_{x}+B f_{y}\right)^{p}=1+$ $p C_{p}^{\text {ext }}\left(A f_{x}+B f_{y}\right)$. Now we consider the relationship between $f_{x}^{p}, f_{y}^{p}$ and the coefficients of $\delta x$ and $\delta y$, respectively. First recall that $n=n^{p}+p \delta(n)$ for any positive integer $n$. So we can write the coefficients of $\delta x$ and $\delta y$ from equation (4.1) as

$$
\begin{aligned}
& \text { Coefficient of } \delta x=-3 x^{2 p}-a_{4}^{p}=-\left(3^{p}+p \delta(3)\right) x^{2 p}-a_{4}^{p} \\
& =-p \delta(3) x^{2 p}+f_{x}^{p}+p C_{p}^{\mathrm{ext}}\left(-3 x^{2}-a_{4}\right) \\
& =f_{x}^{p}+p\left(-\delta(3) x^{2 p}+C_{p}^{\mathrm{ext}}\left(-3 x^{2}-a_{4}\right)\right), \\
& \text { Coefficient of } \delta y=2 y^{p}=\left(2^{p}+p \delta(2)\right) y^{p} \\
& =f_{y}^{p}+p \delta(2) y^{p} \text {. }
\end{aligned}
$$


Then combining these with the equation $A^{p} f_{x}^{p}+B^{p} f_{y}^{p}=1+p C_{p}^{\operatorname{ext}}\left(A f_{x}+B f_{y}\right)$,

$$
\begin{aligned}
A^{p}( & \left.-3 x^{2 p}-a_{4}^{p}\right)+B^{p}\left(2 y^{p}\right) \\
& =A^{p}\left(f_{x}^{p}+p\left(-\delta(3) x^{2 p}+C_{p}^{\operatorname{ext}}\left(-3 x^{2}-a_{4}\right)\right)\right)+B^{p}\left(f_{y}^{p}+p \delta(2) y^{p}\right) \\
& =1+p\left(C_{p}^{\operatorname{ext}}\left(A f_{x}+B f_{y}\right)+A^{p}\left(-\delta(3) x^{2 p}+C_{p}^{\operatorname{ext}}\left(-3 x^{2}-a_{4}\right)\right)+B^{p} \delta(2) y^{p}\right) .
\end{aligned}
$$

Now if we let $R_{U, 0}=C_{p}^{\text {ext }}\left(A f_{x}+B f_{y}\right)+A^{p}\left(-\delta(3) x^{2 p}+C_{p}^{\text {ext }}\left(-3 x^{2}-a_{4}\right)\right)+B^{p} \delta(2) y^{p}$, then $A^{p}\left(-3 x^{2 p}-a_{4}^{p}\right)+B^{p}\left(2 y^{p}\right)=1+p R_{U, 0}$.

With the computations in the previous paragraph we now have enough tools to perform the iteration step in Hensel's Lemma. We assume that

$$
\begin{aligned}
& \delta x=-P_{U, 0} A^{p}+p \eta, \\
& \delta y=-P_{U, 0} B^{p}+p \sigma,
\end{aligned}
$$

and plug these assumptions into equation (4.1). Then we solve the resulting equation for $\eta$ and $\sigma$, keeping in mind that we are working modulo $p^{2}$. (Note: The procedure is the same working modulo $p^{3}$ etc., but in that case one must assume a $p^{2}$ term for the $\delta x$ and $\delta y$ and then perform the iteration twice.)

$$
\begin{gathered}
\left(-3 x^{p} \delta x^{2}-\delta a_{4} \delta x+\delta y^{2}\right) p+\left(-3 x^{2 p}-a_{4}^{p}\right) \delta x+2 y^{p} \delta y+P_{U, 0} \\
=p\left(-3 x^{p} P_{U, 0}^{2} A^{2 p}+\delta a_{4} P_{U, 0} A^{p}+P_{U, 0}^{2} B^{2 p}\right. \\
\left.-P_{U, 0} R_{U, 0}+\left(-3 x^{2 p}-a_{4}^{p}\right) \eta+2 y^{p} \sigma\right) .
\end{gathered}
$$

Now if we let $P_{U, 1}=-3 x^{p} P_{U, 0}^{2} A^{2 p}+\delta a_{4} P_{U, 0} A^{p}+P_{U, 0}^{2} B^{2 p}-P_{U, 0} R_{U, 0}$, then

$$
\begin{gathered}
\eta=-P_{U, 1} A^{p}, \\
\sigma=-P_{U, 1} B^{p} .
\end{gathered}
$$

So the morphism that takes $x$ to $x, y$ to $y, \delta x$ to $-P_{U, 0} A^{p}-p P_{U, 1} A^{p}$, and $\delta y$ to $-P_{U, 0} B^{p}-p P_{U, 1} B^{p}$ will map $\delta f$ to 0 . Our corresponding section, $s_{U}$, is

$$
\left(x, y,-A^{p}\left(P_{U, 0}+p P_{U, 1}\right),-B^{p}\left(P_{U, 0}+p P_{U, 1}\right)\right) .
$$

Next we find the section $s_{V}$ that defines a specific map from $V$ to $V^{1}$ such that under this map $\delta f(z, 1, w)$ is taken to 0 . Since the techniques used are identical to those used to find $s_{U}$, we will omit most of the details. From now on for convenience of notation we will refer to $f(z, 1, w)$ as $g$, and $\frac{\partial g}{\partial z}, \frac{\partial g}{\partial w}$ will be referred to as $g_{z}$ and $g_{w}$, respectively.

Let $P_{V, 0}=-\delta a_{6} w^{3 p}-\delta a_{4} z^{p} w^{2 p}+C_{p}^{\text {ext }}\left(w-z^{3}-a_{4} z w^{2}-a_{6} w^{3}\right)$ and let $C$ and $D$ be polynomials in $M^{0}[z, w]$ such that $C g_{z}+D g_{w}=1$. Specifically

$$
C=z\left(-\frac{3}{2} a_{6} w-a_{4} z\right), \quad D=-\frac{3}{2} a_{6} w^{2}-w a_{4} z+1
$$


Next let

$$
\begin{aligned}
R_{V, 0}= & C_{p}^{\mathrm{ext}}\left(C g_{z}+D g_{w}\right)+C^{p}\left(-\delta(3) z^{2 p}+C_{p}^{\mathrm{ext}}\left(-3 z^{2}-a_{4} w^{2}\right)\right) \\
& +D^{p}\left(-\delta(3) a_{6}^{p} w^{2 p}-\delta(2) a_{4}^{p} z^{p} w^{p}+C_{p}^{\mathrm{ext}}\left(1-3 a_{6} w^{2}-2 a_{4} z w\right)\right)
\end{aligned}
$$

and let

$$
\begin{aligned}
P_{V, 1}= & -3 z^{p}\left(P_{V, 0} C^{p}\right)^{2}+\left(2 a_{4}^{p} w^{p}\left(-P_{V, 0} D^{p}\right)+\delta a_{4} w^{2 p}\right)\left(P_{V, 0} C^{p}\right) \\
& -\left(a_{4}^{p} z^{p}+3 a_{6}^{p} w^{p}\right)\left(P_{V, 0} D^{p}\right)^{2}-\left(3 \delta a_{6} w^{2 p}+2 \delta a_{4} z^{p} w^{p}\right)\left(-P_{V, 0} D^{p}\right)-P_{V, 0} R_{V, 0} .
\end{aligned}
$$

Then the section $s_{V}$ defining a map from $V$ to $V^{1}$ is

$$
\left(z, w,-C^{p}\left(P_{V, 0}+p P_{V, 1}\right),-D^{p}\left(P_{V, 0}+p P_{V, 1}\right)\right) .
$$

\section{5. $s_{U}-s_{V}$ UNDER THE GROUP LAW}

We now need the $\delta z$ coordinate also referred to as $\zeta$ in the difference, $s_{U}-s_{V}$, of these two sections under the group law. We will work with the element $\left(z, w, z^{\prime}, w^{\prime}\right)$ where $z^{\prime}=\delta z$ and $w^{\prime}=\delta w$. Recall from the Introduction that our gluing maps on the intersection $U^{1} \cap V^{1}$ are

$$
\begin{aligned}
z & =-x / y, \\
w & =-1 / y, \\
\delta z & =\frac{x^{p} \delta y-y^{p} \delta x}{y^{p}\left(y^{p}+p \delta y\right)}, \\
\delta w & =\frac{\delta y}{y^{p}\left(y^{p}+p \delta y\right)} .
\end{aligned}
$$

So if we let $x^{\prime}=\delta x$ and $y^{\prime}=\delta y$, then in terms of the coordinates on $U^{1}$, our element is $\left(-x / y,-1 / y, \frac{x^{p} y^{\prime}-y^{p} x^{\prime}}{y^{p}\left(y^{p}+p y^{\prime}\right)}, \frac{y^{\prime}}{y^{p}\left(y^{p}+p y^{\prime}\right)}\right)$, which modulo $p^{2}$ is the same as $\left(-x / y,-1 / y, \frac{1}{y^{3 p}}\left(x^{p} y^{\prime}-y^{p} x^{\prime}\right)\left(y^{p}-p y^{\prime}\right), \frac{1}{y^{3 p}}\left(y^{\prime}\right)\left(y^{p}-p y^{\prime}\right)\right)$. Then under the map $s_{U}$, this element is mapped to

$$
\left(-x / y,-1 / y, \frac{-x^{p} B^{p}\left(P_{U, 0}+p P_{U, 1}\right)+y^{p} A^{p}\left(P_{U, 0}+p P_{U, 1}\right)}{y^{p}\left(y^{p}-p B^{p}\left(P_{U, 0}+p P_{U, 1}\right)\right)}, \frac{-B^{p}\left(P_{U, 0}+p P_{U, 1}\right)}{y^{p}\left(y^{p}-p B^{p}\left(P_{U, 0}+p P_{U, 1}\right)\right)}\right)
$$

which simplifies modulo $p^{2}$ to

$$
\begin{gathered}
\left(-x / y,-1 / y, \frac{\left(-x^{p} B^{p}+y^{p} A^{p}\right)\left(y^{p} P_{U, 0}+p\left(B^{p} P_{U, 0}^{2}+y^{p} P_{U, 1}\right)\right)}{y^{3 p}},\right. \\
\left.\frac{-B^{p}\left(y^{p} P_{U, 0}+p\left(B^{p} P_{U, 0}^{2}+y^{p} P_{U, 1}\right)\right)}{y^{3 p}}\right) .
\end{gathered}
$$

Under the map $s_{V}$ the element $\left(z, w, z^{\prime}, w^{\prime}\right)$ is mapped to

$$
\left(z, w,-C^{p}\left(P_{V, 0}+p P_{V, 1}\right),-D^{p}\left(P_{V, 0}+p P_{V, 1}\right)\right) .
$$

The image of the element $\left(z, w, z^{\prime}, w^{\prime}\right)$ under the difference map $s_{U}-s_{V}$ is the difference under the group law on $E^{1}$ of the image of $\left(z, w, z^{\prime}, w^{\prime}\right)$ under $s_{U}$ and the image of $\left(z, w, z^{\prime}, w^{\prime}\right)$ under $s_{V}$. In order to take the difference we must first take the inverse under the group law of the image of $\left(z, w, z^{\prime}, w^{\prime}\right)$ under $s_{V}$, which is

$$
\left(-z,-w, C^{p}\left(P_{V, 0}+p P_{V, 1}\right), D^{p}\left(P_{V, 0}+p P_{V, 1}\right)\right)
$$


and then add this to the image of $\left(z, w, z^{\prime}, w^{\prime}\right)$ under $s_{U}$. Specifically we will let

$$
\begin{aligned}
z_{1} & =-x / y=z, \\
w_{1} & =-1 / y=w, \\
\delta z_{1} & =\frac{\left(-x^{p} B^{p}+y^{p} A^{p}\right)\left(y^{p} P_{U, 0}+p\left(B^{p} P_{U, 0}^{2}+y^{p} P_{U, 1}\right)\right)}{y^{3 p}} \\
& =\left(w^{p} z^{p} B^{p}+w^{p} A^{p}\right)\left(-P_{U, 0}+p\left(w^{p} B^{p} P_{U, 0}^{2}-P_{U, 1}\right)\right), \\
\delta w_{1} & =\frac{-B^{p}\left(y^{p} P_{U, 0}+p\left(B^{p} P_{U, 0}^{2}+y^{p} P_{U, 1}\right)\right)}{y^{3 p}} \\
& =w^{2 p} B^{p}\left(-P_{U, 0}+p\left(w^{p} B^{p} P_{U, 0}^{2}-P_{U, 1}\right)\right), \\
z_{2} & =-z, \\
w_{2} & =-w, \\
\delta z_{2} & =C^{p}\left(P_{V, 0}+p P_{V, 1}\right), \\
\delta w_{2} & =D^{p}\left(P_{V, 0}+p P_{V, 1}\right),
\end{aligned}
$$

and apply the explicit formulation of the group law detailed in Section 3. Also since for the purpose of our computation we only need the $\delta z_{3}$ term, this is the only one we will formulate in detail.

We are going to be analyzing $\frac{1}{\mu^{3 p}}\left(-\mu^{p} \delta \alpha+\alpha^{p} \delta \mu\right)\left(\mu^{p}-p \delta \mu\right)$ with the above terms substituted in for $z_{1}, w_{1}$, etc. When we do this,

$$
\begin{aligned}
& \alpha=0, \\
& \mu=2 w+6 a_{6} w^{3}+6 a_{4} z w^{2}+6 z^{3},
\end{aligned}
$$

which, if we then add $6 f(z, 1, w)$, we have $\mu=8 w=-8 / y$.

Proposition 5.1. The $\zeta=\delta z$ coordinate of $s_{U}-s_{V}$ is

$$
\zeta=\frac{-\delta \alpha}{(8 w)^{p}}+p \frac{\delta \alpha \delta \mu}{(8 w)^{2 p}},
$$

where the above expressions are used for $z_{1}, z_{2}, \delta z_{1}, \delta z_{2}, \delta w_{1}$, etc. in $\delta \alpha$ and $\delta \mu$.

The next step in the computation of $f_{\text {def }}$ is to apply the formal logarithm of the Frobenius twist of the formal group of the elliptic curve to $\zeta$. This is a triviality as mentioned in the Introduction by the following proposition.

Proposition 5.2. Let $\log _{\mathcal{F}_{1}^{\phi^{1}}}(\xi)$ be the formal logarithm of the Frobenius twist of the formal group of the elliptic curve. Then

$$
\log _{\mathcal{F}_{1}^{\phi^{1}}}(\zeta)=\zeta \text { modulo } p^{2} .
$$

Proof. Recall

$$
\log _{\mathcal{F}_{1}^{\phi^{1}}}(\xi):=\xi+\frac{p \phi\left(c_{1}\right)}{2} \xi^{2}+\frac{p^{2} \phi\left(c_{2}\right)}{3} \xi^{3}+\cdots,
$$

where the $c_{i}$ are the coefficients of the power series expansion of the invariant differential [3, p. 127]. From [6, p. 113] we know that the invariant differential

$$
\omega(z)=\left(1+2 a_{4} z^{4}+\cdots\right) d z
$$

and so $c_{1}=0, c_{2}=0, c_{3}=0, c_{4}=2 a_{4} \cdots$. However, the power of $p$ in $p^{n} / n$ is at least 2 for all $n \geq 4$. Hence, modulo $p^{2}$ the power series $\log _{\mathcal{F}_{1}^{\phi^{1}}}(\xi)$ is the identity. 


\section{Residue of THE COHOMOLOGY ClASS}

Recall that any cohomology class in $H^{1}\left(E \otimes M^{1}, \mathcal{O}\right) \simeq H^{1}(E, \mathcal{O}) \otimes M^{1}$ has a representative of the form $\sum a_{n} y^{n}+x \sum b_{n} y^{n}+x^{2} \sum e_{n} y^{n}$. Let us refer to the coefficient $e_{-1}$ in a sum $\sum a_{n} y^{n}+x \sum b_{n} y^{n}+x^{2} \sum e_{n} y^{n}$ as the residue of the sum. The final step in the computation of $f_{\text {jet }}$ modulo $p^{2}$ is to take the residue of $\zeta=\delta z$ coordinate of $s_{U}-s_{V}$, which is a cohomogy class as a result of the above proposition.

While the idea behind taking the residue is simple, namely write $\zeta$ as $\sum a_{n} y^{n}+$ $x \sum b_{n} y^{n}+x^{2} \sum e_{n} y^{n}$ and take the coefficient of $x^{2} / y$ in this sum which is $e_{-1}$, the practice is computationally unfeasible. Instead we break the process of finding the residue of $\zeta$ into parts. The residue map has some useful properties; namely, it is linear and the residue of any function that is regular on $U$ or any function that is regular on $V$ is zero. So we can take the residue of the terms in $\zeta$ and then add together the result to get the residue of $\zeta$.

As a preliminary step to the task of analyzing the residue of the terms of $\zeta=\delta z$, we write the following expressions in both coordinates of $U$ and coordinates of $V$ :

$$
\begin{aligned}
A & =\frac{2^{4}\left(4 a_{4}^{2}+6 x^{2} a_{4}-9 x a_{6}\right)}{\Delta}=\frac{2^{4}\left(4 a_{4}^{2} w^{2}+6 z^{2} a_{4}-9 z w a_{6}\right)}{w^{2} \Delta}, \\
B & =\frac{2^{3}(9 y)\left(2 x a_{4}-3 a_{6}\right)}{\Delta}=-\frac{2^{3}(9)\left(2 z a_{4}-3 w a_{6}\right)}{w^{2} \Delta}, \\
C & =z\left(-\frac{3}{2} a_{6} w-a_{4} z\right)=\frac{x}{y^{2}}\left(-\frac{3}{2} a_{6}-a_{4} x\right) \\
D & =-\frac{3}{2} a_{6} w^{2}-w a_{4} z+1=\frac{1}{y^{2}}\left(x^{3}-\frac{1}{2} a_{6}\right) \\
P_{U, 0} & =\frac{P_{V, 0}}{w^{3 p}} .
\end{aligned}
$$

We also note that $P_{U, 0}$ and $P_{U, 1}$ are regular on $U$, and that $P_{V, 0}, P_{V, 1}$, and $C_{p}^{\text {ext }}\left(3 a_{6} w^{3}+3 a_{6} w^{3}+3 z^{3}+3 z^{3}+a_{4} w^{2} z+a_{4} w^{2} z+w+w+2 a_{4} w^{2} z+2 a_{4} w^{2} z\right)$ are regular on $V$. So as an example the following combinations of taken from $\frac{-\delta \alpha}{(8 w)^{p}}$ are regular on $V$ :

$$
\frac{-p 3^{p} D^{p} C^{p} a_{6}^{p} w^{p} P_{V, 0}^{2}}{8^{p}}, \quad \frac{p 2 w^{p} a_{4}^{p} z^{p} C^{p} P_{V, 1}}{8^{p}}, \quad \frac{-C^{p} P_{V, 0}}{8^{p}}
$$

More examples of regular combinations on $V$, this time taken from $\frac{\delta \alpha \delta \mu}{(8 w)^{2 p}}$, are

$$
\frac{\left(3^{p} w^{3 p} C^{p} a_{6}^{p} P_{V, 0}\right)\left(3\left(3^{p}\right) a_{6}^{p} w^{4 p} B^{p} P_{U, 0}\right)}{(8 w)^{2 p}} \text { and } \frac{\left(-3^{p} w^{4 p} a_{6}^{p} A^{p} P_{U, 0}\right)\left(2\left(3^{p}\right) \delta\left(a_{6}\right) w^{3 p}\right)}{(8 w)^{2 p}}
$$

Since the residue of terms that are regular on either $U$ or $V$ is zero, we can exclude these terms from consideration in computing the residue class of $\zeta$. This leads to the following proposition in which for brevity's sake we let

$$
\Upsilon=C_{p}^{\mathrm{ext}}\left(y^{2}-x^{3}-a_{4} x-a_{6}\right)
$$


Proposition 6.1. The residue of $\zeta$ is equal to the residue of

$$
\begin{gathered}
\left(\frac{\left(1-2^{p}\right) A^{p}}{y^{p}}+\frac{-2 x^{p} B^{p}}{y^{2 p}}+\frac{\left(-3^{p} a_{6}^{p}-2 a_{4}^{p} x^{p}\right) A^{p}}{y^{3 p}}+\frac{\left(-1-2^{p}\right) x^{p} D^{p}}{y^{3 p}}\right) \frac{P_{U, 0}}{8^{p}} \\
+p\left(F _ { 1 } C _ { p } ^ { \text { ext } } \left(3 a_{6} w^{3}+3 a_{6} w^{3}+3 z^{3}+3 z^{3}+a_{4} w^{2} z\right.\right. \\
\left.+a_{4} w^{2} z+w+w+2 a_{4} w^{2} z+2 a_{4} w^{2} z\right) \\
+F_{2} C_{p}^{\text {ext }}\left(A f_{x}+B f_{y}\right)+F_{3} C_{p}^{\text {ext }}\left(-3 x^{2}-a_{4}\right) \\
\left.+F_{4} \Upsilon^{2}+\left(F_{5}+F_{6}+F_{7}\right) \Upsilon+F_{8}\right),
\end{gathered}
$$

where $F_{i}$ are polynomials in $M_{1}^{1}\left[x^{p}, y^{p}, \Upsilon\right]$.

Proof. This is proved by the very precise removal of almost all regular terms using a computer algebra system.

It is now necessary to compute residues of terms whose residue may be nontrivial. Namely, we provide a formula for the residue of $\frac{x^{a}}{y^{b}}$ which we will call $\gamma_{a, b}$. We let $\left(\begin{array}{l}n \\ k\end{array}\right)$ denote the binomial coefficient with the convention that $\left(\begin{array}{l}n \\ k\end{array}\right)=0$ if $k>n$. Then from [5] we know

Proposition 6.2. Let $a$ and $b$ be positive integers. Let $m$ and $n \in\{0,1,2\}$ be integers such that $a=3 m+n$. Then the residue of $\frac{x^{a}}{y^{b}}$ is

$\gamma_{a, b}=\left\{\begin{array}{l}0 \\ \sum_{k=0}^{\infty}\left(\begin{array}{c}m+k \\ 3 k+2-n\end{array}\right)\left(\begin{array}{c}m-2 k-2+n \\ \frac{b-1}{2}\end{array}\right)(-1)^{m+k-\frac{b-1}{2}}\left(a_{4}\right)^{3 k+2-n}\left(a_{6}\right)^{m-2 k-2+n-\frac{b-1}{2}} \\ \text { if } b \text { is odd. }\end{array}\right.$

Obviously, because of the convention for binomial coefficients, there will be integers $a$ and $b$ with $b$ odd for which $\gamma_{a, b}$ is 0 . In fact, if $\frac{3 b}{2}>a, \gamma_{a, b}=0$ because of the binomial coefficient $\left(\begin{array}{c}m-2 k-2+n \\ \frac{b-1}{2}\end{array}\right)$. We now introduce a series of propositions that are just expanded formulas for expressions found in Proposition 6.1.

\section{Proposition 6.3.}

$$
\begin{aligned}
\Upsilon & =C_{p}^{\operatorname{ext}}\left(y^{2}-x^{3}-a_{4} x-a_{6}\right) \\
& =\frac{1}{p}\left[\left(\sum_{k=1}^{p-1}\left(\begin{array}{l}
p \\
k
\end{array}\right)(-1)^{k}\right) y^{2 p}-\sum_{k=1}^{p-1}\left(\begin{array}{l}
p \\
k
\end{array}\right) x^{3 k}\left(a_{4} x+a_{6}\right)^{p-k}-\sum_{k=1}^{p-1}\left(\begin{array}{l}
p \\
k
\end{array}\right) a_{4}^{k} a_{6}^{p-k} x^{k}\right] .
\end{aligned}
$$

\section{Proposition 6.4.}

$$
\begin{aligned}
& A^{p}=\frac{2^{4 p}\left(4 a_{4}^{2}+6 x^{2} a_{4}-9 x a_{6}\right)^{p}}{\Delta^{p}} \\
& =\frac{2^{4 p}}{\Delta^{p}}\left[4^{p} a_{4}^{2 p}+6^{p} x^{2 p} a_{4}^{p}-9^{p} x^{p} a_{6}^{p}\right. \\
& \left.+\sum_{k=1}^{p-1}\left(\begin{array}{l}
p \\
k
\end{array}\right)\left(4 a_{4}^{2}\right)^{p-k}\left(6 x^{2} a_{4}-9 x a_{6}\right)^{k}+\sum_{k=1}^{p-1}\left(\begin{array}{l}
p \\
k
\end{array}\right)\left(6 x^{2} a_{4}\right)^{k}\left(-9 x a_{6}\right)^{p-k}\right] .
\end{aligned}
$$




\section{Proposition 6.5.}

$$
\begin{aligned}
B^{p} & =\frac{2^{3 p}(9 y)^{p}\left(2 x a_{4}-3 a_{6}\right)^{p}}{\Delta^{p}} \\
& =\frac{2^{3 p}(9 y)^{p}}{\Delta^{p}}\left[2^{p} x^{p} a_{4}^{p}-3^{p} a_{6}^{p}+\sum_{k=1}^{p-1}\left(\begin{array}{l}
p \\
k
\end{array}\right)\left(2 x a_{4}\right)^{k}\left(-3 a_{6}\right)^{p-k}\right] .
\end{aligned}
$$

\section{Proposition 6.6.}

$$
\begin{array}{r}
C_{p}^{\mathrm{ext}}\left(3 a_{6} w^{3}+3 a_{6} w^{3}+3 z^{3}+3 z^{3}+a_{4} w^{2} z+a_{4} w^{2} z+w+w+2 a_{4} w^{2} z+2 a_{4} w^{2} z\right) \\
=\frac{1}{p}\left[2\left(3 a_{6}\right)^{p}+2\left(3 x^{3}\right)^{p}+2\left(1+2^{p}\right)\left(a_{4} x\right)^{p}+\left(2-8^{p}\right) y^{2 p}\right]\left(\frac{-1}{y^{3 p}}\right) .
\end{array}
$$

\section{Proposition 6.7.}

$$
\begin{aligned}
& C_{p}^{\mathrm{ext}}\left(A f_{x}+B f_{y}\right) \\
&=\frac{1}{p} \sum_{k=1}^{p-1} \sum_{i=0}^{k} \sum_{j=0}^{p-i}\left(\begin{array}{c}
p \\
k
\end{array}\right)\left(\begin{array}{c}
k \\
i
\end{array}\right)\left(\begin{array}{c}
p-i \\
j
\end{array}\right)(-1)^{k-i} \\
& \times\left(\frac{9\left(2^{4}\right)}{\Delta}\right)^{p-i}\left(2 a_{4}\right)^{j}\left(-3 a_{6}\right)^{p-i-j} x^{j} y^{2(p-i)} .
\end{aligned}
$$

\section{Proposition 6.8.}

$$
C_{p}^{\text {ext }}\left(-3 x^{2}-a_{4}\right)=-\frac{1}{p} \sum_{k=1}^{p-1}\left(\begin{array}{l}
p \\
k
\end{array}\right) 3^{k} a_{4}^{p-k} x^{2 k} .
$$

Using this series of propositions, we can now explicitly write down the residue of $\zeta$ by computing the residue of the formula in Proposition 6.1. First we introduce some more notation.

Definition 6.9. Define $\mu_{a, b}$ to be the residue of $\frac{x^{a} \Upsilon}{y^{b}}$ where

$$
\Upsilon=C_{p}^{\mathrm{ext}}\left(y^{2}-x^{3}-a_{4} x-a_{6}\right)
$$

Definition 6.10. Define $\tau_{a, b}$ to be the residue of $\frac{x^{a} \Upsilon^{2}}{y^{b}}$ where

$$
\Upsilon=C_{p}^{\mathrm{ext}}\left(y^{2}-x^{3}-a_{4} x-a_{6}\right)
$$

Using the formulas above, it is easy to check that for some, but not all, values, $\mu_{a, b}$ will be zero. Similarly there are values of $a$ and $b$ for which $\tau_{a, b}$ is nonzero and for which it is zero. Some examples of $\mu_{a, b}$ are

$$
\begin{gathered}
\mu_{p, 3 p}=0 \\
\mu_{p, p}=\frac{1}{p}\left[-\sum_{k=1}^{p-1}\left(\begin{array}{l}
p \\
k
\end{array}\right) \sum_{i=0}^{p-k}\left(\begin{array}{c}
p-k \\
i
\end{array}\right) a_{4}^{i} a_{6}^{p-k-i} \gamma_{p+3 k+i, p}-\sum_{k=1}^{p-1}\left(\begin{array}{l}
p \\
k
\end{array}\right) a_{4}^{k} a_{6}^{p-k} \gamma_{p+k, p}\right] .
\end{gathered}
$$

Note that both $\gamma_{a, b}$ and $\mu_{a, b}$ are in $M_{1}^{0}$. We can now prove the following theorem. 
Theorem 6.11. The reduction modulo $p^{2}$ of $f_{\text {jet }}$ is

$$
\begin{aligned}
& {\left[\frac{9^{p}\left(2^{p}-4^{p}-2\left(3^{p}\right)\right) a_{6}^{p} \delta\left(a_{4}\right)}{\Delta^{p}}+\frac{2^{p}\left(-6^{p}+12^{p}+2\left(9^{p}\right)\right) a_{4}^{p} \delta\left(a_{6}\right)}{\Delta^{p}}\right] \gamma_{2 p, p}} \\
& \quad+\frac{1}{\Delta^{p}}\left[2^{p}\left(1-2^{p}\right) 4^{p} a_{4}^{2 p} \mu_{0, p}+\left(-18^{p}\left(1-2^{p}\right)+2\left(27^{p}\right)\right) a_{6}^{p} \mu_{p, p}\right. \\
& \left.\quad+\left(12^{p}\left(1-2^{p}\right)-2\left(18^{p}\right)\right) a_{4}^{p} \mu_{2 p, p}+\left(2\left(18^{p}\right)-36^{p}\right) a_{4}^{p} a_{6}^{p} \mu_{2 p, 3 p}-2\left(12^{p}\right) a_{4}^{2 p} \mu_{3 p, 3 p}\right] \\
& \quad+p\left(H_{0}+H_{1}+H_{2}+H_{3}+H_{4}+H_{5}+H_{6}+H_{7}+H_{8}\right)
\end{aligned}
$$

where $H_{0}$ is

$$
\begin{aligned}
& \frac{1}{p}\left(( 1 - 2 ^ { p } ) \frac { 2 ^ { p } } { \Delta ^ { p } } \left[-\delta\left(a_{4}\right) 6^{p} a_{4}^{p} \gamma_{3 p, p}+\sum_{k=1}^{p-1}\left(\begin{array}{l}
p \\
k
\end{array}\right)\left(4 a_{4}^{2}\right)^{p-k} \sum_{i=0}^{k}\left(\begin{array}{c}
k \\
i
\end{array}\right)\left(6 a_{4}\right)^{i}\left(-9 a_{6}\right)^{k-i}\right.\right. \\
& \times\left(-\delta\left(a_{4}\right) \gamma_{p+k+i, p}-\delta\left(a_{6}\right) \gamma_{k+i, p}+\mu_{k+i, p}\right) \\
& \left.+\sum_{k=1}^{p-1}\left(\begin{array}{l}
p \\
k
\end{array}\right)\left(6 a_{4}\right)^{k}\left(-9 a_{6}\right)^{p-k}\left(-\delta\left(a_{4}\right) \gamma_{2 p+k, p}-\delta\left(a_{6}\right) \gamma_{p+k, p}+\mu_{p+k, p}\right)\right] \\
& -2 \frac{9^{p}}{\Delta^{p}}\left[-\delta\left(a_{4}\right) 2^{p} a_{4}^{p} \gamma_{3 p, p}\right. \\
& \left.+\sum_{k=1}^{p-1}\left(\begin{array}{l}
p \\
k
\end{array}\right)\left(2 a_{4}\right)^{k}\left(-3 a_{6}\right)^{p-k}\left(-\delta\left(a_{4}\right) \gamma_{2 p+k, p}-\delta\left(a_{6}\right) \gamma_{p+k, p}+\mu_{p+k, p}\right)\right] \\
& -\left(3^{p} a_{6}^{p}\right) \frac{2^{p}}{\Delta^{p}}\left[\sum_{k=1}^{p-1}\left(\begin{array}{l}
p \\
k
\end{array}\right)\left(4 a_{4}^{2}\right)^{p-k} \sum_{i=0}^{k}\left(\begin{array}{c}
k \\
i
\end{array}\right)\left(6 a_{4}\right)^{i}\left(-9 a_{6}\right)^{k-i} \mu_{k+i, 3 p}\right. \\
& \left.+\sum_{k=1}^{p-1}\left(\begin{array}{l}
p \\
k
\end{array}\right)\left(6 a_{4}\right)^{k}\left(-9 a_{6}\right)^{p-k} \mu_{p+k, 3 p}\right] \\
& -\left(2 a_{4}^{p}\right) \frac{2^{p}}{\Delta^{p}}\left[\sum_{k=1}^{p-1}\left(\begin{array}{l}
p \\
k
\end{array}\right)\left(4 a_{4}^{2}\right)^{p-k} \sum_{i=0}^{k}\left(\begin{array}{c}
k \\
i
\end{array}\right)\left(6 a_{4}\right)^{i}\left(-9 a_{6}\right)^{k-i} \mu_{p+k+i, 3 p}\right. \\
& \left.\left.+\sum_{k=1}^{p-1}\left(\begin{array}{l}
p \\
k
\end{array}\right)\left(6 a_{4}\right)^{k}\left(-9 a_{6}\right)^{p-k} \mu_{2 p+k, 3 p}\right]\right),
\end{aligned}
$$

$H_{1}$ is

$$
\begin{aligned}
& \frac{-1}{p}\left(9 \delta\left(a_{4}\right) a_{4}^{p}\left(3^{p}\right) \gamma_{3 p, p}\right. \\
& \quad-\frac{9}{2} a_{4}^{p}\left(2\left(3 a_{6}\right)^{p} \mu_{2 p, 3 p}+2\left(3^{p}\right) \mu_{5 p, 3 p}+2\left(1+2^{p}\right) a_{4}^{p} \mu_{3 p, 3 p}+\left(2-8^{p}\right) \mu_{2 p, p}\right) \\
& \quad+\left(9 a_{6}^{p}-\frac{3}{2} a_{4}^{p}\right)\left(2\left(3^{p}\right) \mu_{4 p, 3 p}+2\left(1+2^{p}\right) a_{4}^{p} \mu_{2 p, 3 p}+\left(2-8^{p}\right) \mu_{p, p}\right) \\
& \quad-a_{4}^{2 p}\left(2\left(3^{p}\right) \mu_{3 p, 3 p}+\left(2-8^{p}\right) \mu_{0, p}\right) \\
& +\left(\left(\frac{3}{2} a_{4}^{p}-9 a_{6}^{p}\right) \delta\left(a_{4}\right)+\frac{9}{2} a_{4}^{p} \delta\left(a_{6}\right)\right)\left(2\left(3^{p}\right) \gamma_{5 p, 3 p}+\left(2-8^{p}\right) \gamma_{2 p, p}\right) \\
& \left.\quad+\left(-3 a_{4}^{2 p}+\frac{9}{2} a_{6}^{p} a_{4}^{p}\right)\left(2\left(3^{p}\right) \mu_{5 p, 5 p}+\left(2-8^{p}\right) \mu_{2 p, 3 p}\right)\right) / \Delta^{p}
\end{aligned}
$$


$\mathrm{H}_{2}$ is

$$
\begin{gathered}
\frac{1}{p} \sum_{k=1}^{p-1} \sum_{i=0}^{k} \sum_{j=0}^{p-i}\left(\begin{array}{c}
p \\
k
\end{array}\right)\left(\begin{array}{c}
k \\
i
\end{array}\right)\left(\begin{array}{c}
p-i \\
j
\end{array}\right)(-1)^{k-i}\left(\frac{9\left(2^{4}\right)}{\Delta}\right)^{p-i}\left(2 a_{4}\right)^{j}\left(-3 a_{6}\right)^{p-i-j} \\
\times\left[\left(\left(24 a_{4}^{2 p}-36 a_{6}^{p} a_{4}^{p}\right) \mu_{2 p+j, 3 p+2(p-i)}+\left(36 a_{6}^{p} a_{4}^{p}+16 a_{4}^{3 p}-54 a_{6}^{2 p}\right) \mu_{p+j, 3 p+2(p-i)}\right.\right. \\
+24 a_{6}^{p} a_{4}^{2 p} \mu_{j, 3 p+2(p-i)}+36 a_{4}^{p} \mu_{2 p+j, p+2(p-i)} \\
\left.+\left(12 a_{4}^{p}-72 a_{6}^{p}\right) \mu_{p+j, p+2(p-i)}+8 a_{4}^{2 p} \mu_{j, p+2(p-i)}\right) / \Delta^{p} \\
+\left(36 a_{4}^{p} \delta\left(a_{4}\right) \gamma_{3 p+j, p+2(p-i)}\right. \\
+\left(\left(72 a_{6}^{p}-12 a_{4}^{p}\right) \delta\left(a_{4}\right)-36 a_{4}^{p} \delta\left(a_{6}\right)\right) \gamma_{2 p+j, p+2(p-i)} \\
+\left(28 a_{4}^{2 p} \delta\left(a_{4}\right)+\left(72 a_{6}^{p}-12 a_{4}^{p}\right) \delta\left(a_{6}\right)\right) \gamma_{p+j, p+2(p-i)} \\
\left.+\left(\left(72 a_{4}^{p} a_{6}^{p}-24 a_{4}^{2 p}\right) \delta\left(a_{4}\right)-8 a_{4}^{2 p} \delta\left(a_{6}\right)\right) \gamma_{j, p+2(p-i)}\right) / \Delta^{p} \\
+\left(\left(\left(54 a_{6}^{2 p}-16 a_{4}^{3 p}-36 a_{4}^{p} a_{6}^{p}\right) \delta\left(a_{4}\right)+\left(36 a_{4}^{p} a_{6}^{p}-24 a_{4}^{2 p}\right) \delta\left(a_{6}\right)\right) \gamma_{2 p+j, 3 p+2(p-i)}\right. \\
+\left(\left(24 a_{4}^{3 p}-60 a_{4}^{2 p} a_{6}^{p}\right) \delta\left(a_{4}\right)\right. \\
\left.\left.\left.+\left(54 a_{6}^{2 p}-16 a_{4}^{3 p}-36 a_{4}^{p} a_{6}^{p}\right) \delta\left(a_{6}\right)\right) \gamma_{p+j, 3 p+2(p-i)}\right) / \Delta^{p}\right]
\end{gathered}
$$

$\mathrm{H}_{3}$ is

$$
\begin{aligned}
& -\frac{1}{p} \sum_{k=1}^{p-1}\left(\begin{array}{l}
p \\
k
\end{array}\right) 3^{k} a_{4}^{p-k} \\
& \times\left(\left(\left(2304 a_{4}^{3 p}+10368 a_{6}^{2 p}-8640 a_{6}^{p} a_{4}^{p}+1152 a_{4}^{2 p}\right) \mu_{2 p+2 k, p}\right.\right. \\
& +\left(-1920 a_{4}^{3 p}-576 a_{6}^{p} a_{4}^{2 p}\right) \mu_{p+2 k, p} \\
& +\left(512 a_{4}^{4 p}+10368 a_{6}^{2 p} a_{4}^{p}-10368 a_{6}^{p} a_{4}^{2 p}+2304 a_{4}^{3 p}\right) \mu_{2 k, p} \\
& +\left(-10368 a_{6}^{2 p} a_{4}^{p}+3456 a_{6}^{p} a_{4}^{2 p}\right. \\
& \left.+3072 a_{4}^{4 p}+7776 a_{6}^{3 p}-4608 a_{6}^{p} a_{4}^{3 p}\right) \mu_{2 p+2 k, 3 p} \\
& +\left(11520 a_{6}^{p} a_{4}^{3 p}+1024 a_{4}^{5 p}-12096 a_{6}^{2 p} a_{4}^{2 p}-2304 a_{4}^{4 p}\right) \mu_{p+2 k, 3 p} \\
& \left.+\left(-5184 a_{6}^{3 p} a_{4}^{p}-2304 a_{6}^{p} a_{4}^{3 p}+1536 a_{6}^{p} a_{4}^{4 p}+6912 a_{6}^{2 p} a_{4}^{2 p}\right) \mu_{2 k, 3 p}\right) / \Delta^{2 p} \\
& +\left(\left(\left(1920 a_{4}^{3 p}+576 a_{6}^{p} a_{4}^{2 p}\right) \delta\left(a_{4}\right)\right.\right. \\
& \left.+\left(8640 a_{6}^{p} a_{4}^{p}-1152 a_{4}^{2 p}-2304 a_{4}^{3 p}-10368 a_{6}^{2 p}\right) \delta\left(a_{6}\right)\right) \gamma_{2 p+2 k, p} \\
& +\left(\left(1792 a_{4}^{4 p}-1152 a_{4}^{3 p}+1728 a_{6}^{p} a_{4}^{2 p}\right) \delta\left(a_{4}\right)\right. \\
& \left.+\left(1920 a_{4}^{3 p}+576 a_{6}^{p} a_{4}^{2 p}\right) \delta\left(a_{6}\right)\right) \gamma_{p+2 k, p} \\
& +\left(\left(6912 a_{6}^{p} a_{4}^{3 p}+1728 a_{6}^{2 p} a_{4}^{p}\right.\right. \\
& \left.+2592 a_{6}^{3 p}-3072 a_{4}^{4 p}-2304 a_{6}^{p} a_{4}^{2 p}\right) \delta\left(a_{4}\right) \\
& \left.\left.\left.+\left(-512 a_{4}^{4 p}-10368 a_{6}^{2 p} a_{4}^{p}+10368 a_{6}^{p} a_{4}^{2 p}-2304 a_{4}^{3 p}\right) \delta\left(a_{6}\right)\right) \gamma_{2 k, p}\right) / \Delta^{2 p}\right),
\end{aligned}
$$


$\mathrm{H}_{4}$ is

$$
\begin{aligned}
& \left(-\frac{1}{2}\left(-3732480 a_{6}^{2 p} a_{4}^{2 p}-829440 a_{6}^{p} a_{4}^{4 p}\right.\right. \\
& +3359232 a_{6}^{3 p} a_{4}^{p}+3456 a_{4}^{2 p} \Delta^{p}+774144 a_{4}^{5 p} \\
& \left.+1990656 a_{6}^{p} a_{4}^{3 p}-12960 a_{4}^{p} a_{6}^{p} \Delta^{p}-663552 a_{4}^{4 p}\right) \tau_{2 p, p} \\
& -\frac{1}{2}\left(2592 a_{4}^{2 p} \Delta^{p}-3317760 a_{4}^{5 p}-7713792 a_{6}^{2 p} a_{4}^{3 p}\right. \\
& +19440 a_{6}^{2 p} \Delta^{p}+9123840 a_{6}^{p} a_{4}^{4 p}-7278336 a_{6}^{4 p} \\
& +2985984 a_{6}^{2 p} a_{4}^{2 p}-1327104 a_{6}^{p} a_{4}^{3 p}+3359232 a_{6}^{3 p} a_{4}^{p} \\
& \left.+24576 a_{4}^{6 p}-12960 a_{4}^{p} a_{6}^{p} \Delta^{p}-288 a_{4}^{3 p} \Delta^{p}\right) \tau_{p, p} \\
& -\frac{1}{2}\left(-1769472 a_{4}^{6 p}-8957952 a_{6}^{3 p} a_{4}^{2 p}-11232 a_{4}^{2 p} a_{6}^{p} \Delta^{p}\right. \\
& +2875392 a_{4}^{5 p} a_{6}^{p}+3456 a_{4}^{3 p} \Delta^{p}+1327104 a_{4}^{5 p} \\
& \left.\left.+20404224 a_{6}^{2 p} a_{4}^{3 p}-10616832 a_{6}^{p} a_{4}^{4 p}\right) \tau_{0, p}\right) /\left(\Delta^{3 p}\right) \\
& +\left(-\frac{1}{2}\left(-393216 a_{4}^{7 p}+22394880 a_{6}^{3 p} a_{4}^{2 p}-3456 a_{4}^{2 p} a_{6}^{p} \Delta^{p}\right.\right. \\
& -11197440 a_{6}^{4 p} a_{4}^{p}-10616832 a_{4}^{5 p} a_{6}^{p}+2592 a_{4}^{p} a_{6}^{2 p} \Delta^{p} \\
& +3317760 a_{6}^{p} a_{4}^{4 p}+1152 a_{4}^{4 p} \Delta^{p}+2654208 a_{4}^{6 p} \\
& \left.-14929920 a_{6}^{2 p} a_{4}^{3 p}+9953280 a_{6}^{2 p} a_{4}^{4 p}+1152 a_{4}^{3 p} \Delta^{p}\right) \tau_{2 p, 3 p} \\
& -\frac{1}{2}\left(12607488 a_{4}^{5 p} a_{6}^{p}-8957952 a_{6}^{3 p} a_{4}^{2 p}-3244032 a_{6}^{p} a_{4}^{6 p}\right. \\
& +19408896 a_{6}^{3 p} a_{4}^{3 p}+1536 a_{4}^{4 p} \Delta^{p}+13436928 a_{6}^{4 p} a_{4}^{p} \\
& -28864512 a_{6}^{2 p} a_{4}^{4 p}+5184 a_{4}^{2 p} a_{6}^{p} \Delta^{p}+11664 a_{6}^{3 p} \Delta^{p} \\
& -1327104 a_{4}^{6 p}-15552 a_{4}^{p} a_{6}^{2 p} \Delta^{p}+1769472 a_{4}^{7 p} \\
& \left.-2304 a_{4}^{3 p} a_{6}^{p} \Delta^{p}+1990656 a_{6}^{2 p} a_{4}^{3 p}-6718464 a_{6}^{5 p}\right) \tau_{p, 3 p} \\
& -\frac{1}{2}\left(-20901888 a_{6}^{3 p} a_{4}^{3 p}-18144 a_{4}^{2 p} a_{6}^{2 p} \Delta^{p}-3456 a_{4}^{4 p} \Delta^{p}\right. \\
& +9953280 a_{6}^{2 p} a_{4}^{4 p}+13436928 a_{6}^{4 p} a_{4}^{2 p}+17280 a_{4}^{3 p} a_{6}^{p} \Delta^{p} \\
& +1769472 a_{6}^{p} a_{4}^{6 p}+1664 a_{4}^{5 p} \Delta^{p} \\
& \left.\left.-2654208 a_{6}^{2 p} a_{4}^{5 p}-1327104 a_{4}^{5 p} a_{6}^{p}\right) \tau_{0,3 p}\right) /\left(\Delta^{3 p}\right) \\
& +\left(-\frac{1}{2}\left(-10368 a_{4}^{3 p} a_{6}^{p} \Delta^{p}+31104 a_{4}^{2 p} a_{6}^{2 p} \Delta^{p}-54 \Delta^{2 p} a_{4}^{p}\right.\right. \\
& \left.\left.+10368 \Delta^{p} a_{6}^{p} a_{4}^{4 p}-23328 \Delta^{p} a_{6}^{3 p} a_{4}^{p}-4608 a_{4}^{5 p} \Delta^{p}\right) \tau_{2 p, 5 p}\right) /\left(\Delta^{3 p}\right),
\end{aligned}
$$


$\mathrm{H}_{5}$ is

$$
\begin{gathered}
\left(\left(\frac { 1 } { 2 } \left(-14556672 a_{6}^{4 p}-6635520 a_{4}^{5 p}+6718464 a_{6}^{3 p} a_{4}^{p}+5971968 a_{6}^{2 p} a_{4}^{2 p}\right.\right.\right. \\
+2880 a_{4}^{2 p} \Delta^{p}+18144 a_{6}^{2 p} \Delta^{p}-5184 a_{4}^{3 p} \Delta^{p}-15427584 a_{6}^{2 p} a_{4}^{3 p} \\
\left.-2654208 a_{6}^{p} a_{4}^{3 p}-8640 a_{4}^{p} a_{6}^{p} \Delta^{p}+49152 a_{4}^{6 p}+18247680 a_{6}^{p} a_{4}^{4 p}\right) \delta\left(a_{4}\right) \\
+\frac{1}{2}\left(1548288 a_{4}^{5 p}+6912 a_{4}^{2 p} \Delta^{p}-25920 a_{4}^{p} a_{6}^{p} \Delta^{p}-1658880 a_{6}^{p} a_{4}^{4 p}\right. \\
\left.+6718464 a_{6}^{3 p} a_{4}^{p}-7464960 a_{6}^{2 p} a_{4}^{2 p}+3981312 a_{6}^{p} a_{4}^{3 p}-1327104 a_{4}^{4 p}\right) \delta\left(a_{6}\right) \\
+\frac{1}{2}\left(3584 a_{4}^{4 p} \delta(3) \Delta^{p}+3456 \delta(3) a_{4}^{2 p} a_{6}^{p} \Delta^{p}+4608 \delta(2) a_{4}^{4 p} \Delta^{p}\right. \\
\left.\left.\left.+18 \delta(3) a_{4}^{p} \Delta^{2 p}-2304 a_{4}^{3 p} \delta(3) \Delta^{p}\right)\right) \mu_{2 p, p}\right) /\left(\Delta^{3 p}\right),
\end{gathered}
$$

$H_{6}$ is

$$
\begin{aligned}
& \left(\left(\frac { 1 } { 2 } \left(3840 a_{4}^{3 p} \Delta^{p}-25214976 a_{6}^{p} a_{4}^{4 p}\right.\right.\right. \\
& +3981312 a_{4}^{5 p}+7409664 a_{4}^{5 p} a_{6}^{p}+4608 a_{4}^{2 p} a_{6}^{p} \Delta^{p} \\
& \left.\quad-5087232 a_{4}^{6 p}+48273408 a_{6}^{2 p} a_{4}^{3 p}-24634368 a_{6}^{3 p} a_{4}^{2 p}\right) \delta\left(a_{4}\right) \\
& +\frac{1}{2}\left(-14556672 a_{6}^{4 p}-15427584 a_{6}^{2 p} a_{4}^{3 p}-25920 a_{4}^{p} a_{6}^{p} \Delta^{p}\right. \\
& \quad-2654208 a_{6}^{p} a_{4}^{3 p}-6635520 a_{4}^{5 p}+38880 a_{6}^{2 p} \Delta^{p} \\
& \quad+18247680 a_{6}^{p} a_{4}^{4 p}+5184 a_{4}^{2 p} \Delta^{p}+6718464 a_{6}^{3 p} a_{4}^{p} \\
& \left.+5971968 a_{6}^{2 p} a_{4}^{2 p}-576 a_{4}^{3 p} \Delta^{p}+49152 a_{4}^{6 p}\right) \delta\left(a_{6}\right) \\
& +\frac{1}{2}\left(5184 \delta(3) a_{6}^{3 p} \Delta^{p}-24 \delta(2) a_{4}^{p} \Delta^{2 p}-36 \delta(3) a_{6}^{p} \Delta^{2 p}+23328 \delta(2) a_{6}^{3 p} \Delta^{p}\right. \\
& +36 \delta(2) a_{6}^{p} \Delta^{2 p}-6912 \delta(2) a_{4}^{4 p} \Delta^{p}+12672 a_{4}^{3 p} \delta(3) a_{6}^{p} \Delta^{p} \\
& \quad-15552 \delta(2) a_{6}^{2 p} a_{4}^{p} \Delta^{p}+3456 \delta(3) a_{6}^{2 p} a_{4}^{p} \Delta^{p}+10368 \delta(2) a_{4}^{3 p} a_{6}^{p} \Delta^{p} \\
& \left.\left.\quad-4608 \delta(3) a_{4}^{2 p} a_{6}^{p} \Delta^{p}-9984 a_{4}^{4 p} \delta(3) \Delta^{p}+6 \delta(3) a_{4}^{p} \Delta^{2 p}\right)\right) \mu_{p, p} \\
& +\left(\frac { 1 } { 2 } \left(7962624 a_{6}^{p} a_{4}^{4 p}-33841152 a_{6}^{2 p} a_{4}^{3 p}-22781952 a_{4}^{5 p} a_{6}^{p}\right.\right. \\
& +5308416 a_{4}^{6 p}+52254720 a_{6}^{3 p} a_{4}^{2 p}-29113344 a_{6}^{4 p} a_{4}^{p} \\
& +54 \Delta^{2 p} a_{4}^{p}-786432 a_{4}^{7 p}+6912 a_{4}^{2 p} a_{6}^{p} \Delta^{p}+10368 a_{4}^{p} a_{6}^{2 p} \Delta^{p} \\
& \left.-2304 a_{4}^{3 p} \Delta^{p}+1280 a_{4}^{4 p} \Delta^{p}+21565440 a_{6}^{2 p} a_{4}^{4 p}\right) \delta\left(a_{4}\right) \\
& +\frac{1}{2}\left(40808448 a_{6}^{2 p} a_{4}^{3 p}-22464 a_{4}^{2 p} a_{6}^{p} \Delta^{p}\right. \\
& -17915904 a_{6}^{3 p} a_{4}^{2 p}+5750784 a_{4}^{5 p} a_{6}^{p}+2654208 a_{4}^{5 p} \\
& \left.\quad-21233664 a_{6}^{p} a_{4}^{4 p}-3538944 a_{4}^{6 p}+6912 a_{4}^{3 p} \Delta^{p}\right) \delta\left(a_{6}\right) \\
& +\frac{1}{2}\left(-6912 \delta(2) a_{4}^{3 p} a_{6}^{p} \Delta^{p}-2048 a_{4}^{5 p} \delta(3) \Delta^{p}\right. \\
& +4608 a_{4}^{4 p} \delta(3) \Delta^{p}-14 \delta(3) a_{4}^{2 p} \Delta^{2 p}+23040 \delta(3) a_{6}^{2 p} a_{4}^{2 p} \Delta^{p} \\
& \left.\left.\left.\left.\quad-26880 a_{4}^{3 p} \delta(3) a_{6}^{p} \Delta^{p}+2 \delta(2) a_{4}^{2 p} \Delta^{2 p}\right)\right) \mu_{0, p}\right) /\left(\Delta^{3 p}\right)\right)
\end{aligned}
$$


$H_{7}$ is

$$
\begin{aligned}
\left(\frac{1}{2}(-\right. & 17915904 a_{6}^{3 p} a_{4}^{2 p}+25214976 a_{4}^{5 p} a_{6}^{p}+7776 a_{6}^{3 p} \Delta^{p} \\
& +38817792 a_{6}^{3 p} a_{4}^{3 p}+4608 a_{4}^{3 p} a_{6}^{p} \Delta^{p}+3456 a_{4}^{2 p} a_{6}^{p} \Delta^{p} \\
& +3981312 a_{6}^{2 p} a_{4}^{3 p}+26873856 a_{6}^{4 p} a_{4}^{p}-2654208 a_{4}^{6 p} \\
& -13436928 a_{6}^{5 p}-30 \Delta^{2 p} a_{4}^{p}-6488064 a_{6}^{p} a_{4}^{6 p}+3538944 a_{4}^{7 p} \\
& \left.-36 \Delta^{2 p} a_{6}^{p}-10368 a_{4}^{p} a_{6}^{2 p} \Delta^{p}-3072 a_{4}^{4 p} \Delta^{p}-57729024 a_{6}^{2 p} a_{4}^{4 p}\right) \delta\left(a_{4}\right) \\
+\frac{1}{2} & \left(54 \Delta^{2 p} a_{4}^{p}+5184 a_{4}^{p} a_{6}^{2 p} \Delta^{p}-21233664 a_{4}^{5 p} a_{6}^{p}\right. \\
+ & 19906560 a_{6}^{2 p} a_{4}^{4 p}+2304 a_{4}^{3 p} \Delta^{p}+6635520 a_{6}^{p} a_{4}^{4 p} \\
& -29859840 a_{6}^{2 p} a_{4}^{3 p}+2304 a_{4}^{4 p} \Delta^{p}-6912 a_{4}^{2 p} a_{6}^{p} \Delta^{p} \\
& \left.+44789760 a_{6}^{3 p} a_{4}^{2 p}+5308416 a_{4}^{6 p}-22394880 a_{6}^{4 p} a_{4}^{p}-786432 a_{4}^{7 p}\right) \delta\left(a_{6}\right) \\
+\frac{1}{2} & \left(25920 a_{6}^{3 p} \delta(3) a_{4}^{p} \Delta^{p}+6144 a_{4}^{5 p} \delta(3) \Delta^{p}-36 \delta(2) a_{4}^{p} a_{6}^{p} \Delta^{2 p}\right. \\
+ & 11520 a_{4}^{3 p} \delta(3) a_{6}^{p} \Delta^{p}+6 \delta(2) a_{4}^{2 p} \Delta^{2 p}+6 \delta(3) a_{4}^{2 p} \Delta^{2 p} \\
+ & \left.\left.18 \delta(3) a_{6}^{p} a_{4}^{p} \Delta^{2 p}-34560 \delta(3) a_{6}^{2 p} a_{4}^{2 p} \Delta^{p}-12288 a_{4}^{4 p} \delta(3) a_{6}^{p} \Delta^{p}\right)\right) \mu_{2 p, 3 p} /\left(\Delta^{3 p}\right)
\end{aligned}
$$

and $H_{8}$ is

$$
\begin{aligned}
& \left(-\left(-1271808 a_{4}^{6 p}+995328 a_{4}^{5 p}+12068352 a_{6}^{2 p} a_{4}^{3 p}\right.\right. \\
& \quad-6158592 a_{6}^{3 p} a_{4}^{2 p}+1852416 a_{4}^{5 p} a_{6}^{p} \\
& \left.\quad-6303744 a_{6}^{p} a_{4}^{4 p}+1920 a_{4}^{3 p} \Delta^{p}+1440 a_{4}^{2 p} a_{6}^{p} \Delta^{p}\right) \delta\left(a_{4}\right)^{2} \\
& -\left(9123840 a_{6}^{p} a_{4}^{4 p}-7278336 a_{6}^{4 p}+24576 a_{4}^{6 p}\right. \\
& \quad-3317760 a_{4}^{5 p}-7713792 a_{6}^{2 p} a_{4}^{3 p}-2592 a_{4}^{3 p} \Delta^{p} \\
& \quad+9072 a_{6}^{2 p} \Delta^{p}-4320 a_{4}^{p} a_{6}^{p} \Delta^{p}-1327104 a_{6}^{p} a_{4}^{3 p} \\
& \left.\quad+1440 a_{4}^{2 p} \Delta^{p}+2985984 a_{6}^{2 p} a_{4}^{2 p}+3359232 a_{6}^{3 p} a_{4}^{p}\right) \delta\left(a_{4}\right) \delta\left(a_{6}\right) \\
& -\left(387072 a_{4}^{5 p}+1728 a_{4}^{2 p} \Delta^{p}-1866240 a_{6}^{2 p} a_{4}^{2 p}\right. \\
& \quad+995328 a_{6}^{p} a_{4}^{3 p}-331776 a_{4}^{4 p}+1679616 a_{6}^{3 p} a_{4}^{p} \\
& \left.\quad-6480 a_{4}^{p} a_{6}^{p} \Delta^{p}-414720 a_{6}^{p} a_{4}^{4 p}\right) \delta\left(a_{6}\right)^{2} \\
& -\left(18 \delta(2) a_{6}^{p} \Delta^{2 p}-18 \delta(3) a_{6}^{p} \Delta^{2 p}+1728 \delta(3) a_{6}^{2 p} a_{4}^{p} \Delta^{p}\right. \\
& \quad-7776 \delta(2) a_{6}^{2 p} a_{4}^{p} \Delta^{p}+6336 a_{4}^{3 p} \delta(3) a_{6}^{p} \Delta^{p} \\
& \quad-2304 \delta(3) a_{4}^{2 p} a_{6}^{p} \Delta^{p}+5184 \delta(2) a_{4}^{3 p} a_{6}^{p} \Delta^{p} \\
& \quad+3 \delta(3) a_{4}^{p} \Delta^{2 p}-12 \delta(2) a_{4}^{p} \Delta^{2 p}-3456 \delta(2) a_{4}^{4 p} \Delta^{p} \\
& \left.\quad+11664 \delta(2) a_{6}^{3 p} \Delta^{p}+2592 \delta(3) a_{6}^{3 p} \Delta^{p}-4992 a_{4}^{4 p} \delta(3) \Delta^{p}\right) \delta\left(a_{4}\right) \\
& -\left(1792 a_{4}^{4 p} \delta(3) \Delta^{p}-1152 a_{4}^{3 p} \delta(3) \Delta^{p}+1728 \delta(3) a_{4}^{2 p} a_{6}^{p} \Delta^{p}\right. \\
& \left.\left.\quad+9 \delta(3) a_{4}^{p} \Delta^{2 p}+2304 \delta(2) a_{4}^{4 p} \Delta^{p}\right) \delta\left(a_{6}\right)\right) \frac{\gamma_{2 p, p}}{\Delta^{3 p}} .
\end{aligned}
$$


Proof. We simply apply the most recent propositions to the actual formulas from Proposition 6.1. It should be noted that the $H_{i}$ correspond to the $F_{i}$ in Proposition 6.1 and that upon further analysis certain terms like $\frac{\left(-1-2^{p}\right) x^{p} D^{p}}{y^{3 p}}$ have zero residue even though it is not immediately obvious that the term is regular.

From now on, when we refer to $H_{0}, H_{1}, H_{2}, H_{3}, H_{4}, H_{5}, H_{6}, H_{7}$, and $H_{8}$ we will mean the polynomials in this theorem. We note that $H_{0}, H_{1}, H_{2}, H_{3}, H_{5}, H_{6}, H_{7}$ are in $M_{1}^{1}$ and are linear in $\delta\left(a_{4}\right)$ and $\delta\left(a_{6}\right), H_{4} \in M_{1}^{0}$, and $H_{8} \in M_{1}^{1}$ is quadratic in $\delta\left(a_{4}\right)$ and $\delta\left(a_{6}\right)$.

\section{ORDER TWO MODULAR FORMS}

We remind ourselves that $\phi$, the unique lifting of the Frobenius morphism to $R$, extends to a homomorphism from $M_{1}^{1} \rightarrow M_{1}^{2}$ by taking, e.g., $a_{4} \mapsto a_{4}^{p}+p \delta\left(a_{4}\right)$ and $\delta\left(a_{6}\right) \mapsto \delta\left(a_{6}\right)^{p}+p \delta\left(a_{6}\right)$. Hence if we start with a polynomial in $M^{0}$ like $\gamma_{a, b}$, then $\phi\left(\gamma_{a, b}\right)=\gamma_{a, b}\left(a_{4}^{p}+p \delta\left(a_{4}\right), a_{6}^{p}+p \delta\left(a_{6}\right)\right) \in M^{1}$, where by this notation we mean substitute $a_{4}^{p}+p \delta\left(a_{4}\right)$ in for $a_{4}$ and $a_{6}^{p}+p \delta\left(a_{6}\right)$ in for $a_{6}$.

Definition 7.1. Let $\widetilde{\gamma}_{a, b}$ be the polynomial in $M^{1}$ such that $\phi\left(\gamma_{a, b}\right)=\gamma_{a, b}\left(a_{4}^{p}, a_{6}^{p}\right)+$ $p \widetilde{\gamma}_{a, b}$.

An explicit formula for $\widetilde{\gamma}_{a, b}$ is simple to compute by expanding the formula

$$
\phi\left(\gamma_{a, b}\right)=\left\{\begin{array}{cc}
0 & \text { if } \mathrm{b} \text { is even }, \\
\sum_{k=0}^{\infty}\left(\begin{array}{c}
m+k \\
3 k+2-n
\end{array}\right)\left(\begin{array}{c}
m-2 k-2+n \\
\frac{b-1}{2}
\end{array}\right) & \\
(-1)^{m+k-\frac{b-1}{2}}\left(a_{4}^{p}+p \delta\left(a_{4}\right)\right)^{3 k+2-n}\left(a_{6}^{p}+p \delta\left(a_{6}\right)\right)^{m-2 k-2+n-\frac{b-1}{2}} & \text { if b is odd. }
\end{array}\right.
$$

and modulo $p^{2}, \widetilde{\gamma}_{a, b}$ is linear in $\delta\left(a_{4}\right), \delta\left(a_{6}\right)$. In addition $\phi\left(\gamma_{a, b}\right)=\gamma_{a, b}^{p}+p \delta\left(\gamma_{a, b}\right)$; however, note that $\widetilde{\gamma}_{a, b}$ does not equal $p \delta\left(\gamma_{a, b}\right)$ because the latter is missing the terms from $\gamma_{a, b}^{p}$ whose coefficients are divisible by $p$.

Definition 7.2. Let $\widetilde{\mu}_{a, b}$ be the polynomial in $M^{1}$ such that $\phi\left(\mu_{a, b}\right)=\mu_{a, b}\left(a_{4}^{p}, a_{6}^{p}\right)+$ $p \widetilde{\mu}_{a, b}$.

We recall that the isogeny covariant differential modular form $f_{\text {jet }} h_{\text {jet }}$ is $\phi\left(f_{\text {jet }}\right)$.

Theorem 7.3. The reduction modulo $p^{2}$ of $f_{\text {jet }} h_{\text {jet }}$ is

$$
\begin{aligned}
& {\left[\frac{-72 a_{6}^{p^{2}} \delta\left(a_{4}\right)^{p}+48 a_{4}^{p^{2}} \delta\left(a_{6}\right)^{p}}{\Delta^{p^{2}}}\right] \gamma_{2 p, p}\left(a_{4}^{p}, a_{6}^{p}\right)} \\
& \quad+\frac{1}{\Delta^{p^{2}}}\left[-8 a_{4}^{2 p^{2}} \mu_{0, p}\left(a_{4}^{p}, a_{6}^{p}\right)+72 a_{6}^{p^{2}} \mu_{p, p}\left(a_{4}^{p}, a_{6}^{p}\right)\right. \\
& \left.\quad-48 a_{4}^{p^{2}} \mu_{2 p, p}\left(a_{4}^{p}, a_{6}^{p}\right)-24 a_{4}^{2 p^{2}} \mu_{3 p, 3 p}\left(a_{4}^{p}, a_{6}^{p}\right)\right] \\
& \quad+p\left[\frac{-72 a_{6}^{p^{2}} \delta^{2}\left(a_{4}\right)+48 a_{4}^{p^{2}} \delta^{2}\left(a_{6}\right)}{\Delta^{p^{2}}}\right] \gamma_{2 p, p}\left(a_{4}^{p}, a_{6}^{p}\right)+p J_{0},
\end{aligned}
$$


where $J_{0}$ is

$$
\begin{aligned}
\left(\frac{1}{\Delta^{p^{2}}}[(\right. & \left.-72 a_{6}^{p^{2}} \delta\left(a_{4}\right)^{p}+48 a_{4}^{p^{2}} \delta\left(a_{6}\right)^{p}\right) \widetilde{\gamma}_{2 p, p}-8 a_{4}^{2 p^{2}} \widetilde{\mu}_{0, p} \\
& +72 a_{6}^{p^{2}} \widetilde{\mu}_{p, p}-48 a_{4}^{p^{2}} \widetilde{\mu}_{2 p, p}-24 a_{4}^{2 p^{2}} \widetilde{\mu}_{3 p, 3 p} \\
& +\left((27 \delta(2)+66 \delta(3)) a_{6}^{p^{2}} \delta\left(a_{4}\right)^{p}\right. \\
+ & \left.(-28 \delta(3)-42 \delta(2)) a_{4}^{p^{2}} \delta\left(a_{6}\right)^{p}\right) \gamma_{2 p, p}^{p}+20 \delta(2)^{p} a_{4}^{2 p^{2}} \mu_{0, p}^{p} \\
+ & (-27 \delta(2)-66 \delta(3)) a_{6}^{p^{2}} \mu_{p, p}^{p}+(42 \delta(2)+28 \delta(3)) a_{4}^{p^{2}} \mu_{2 p, p}^{p} \\
& \left.+18 \delta(2) a_{4}^{p^{2}} a_{6}^{p^{2}} \mu_{2 p, 3 p}^{p}+(8 \delta(3)+24 \delta(2)) a_{4}^{2 p^{2}} \mu_{3 p, 3 p}^{p}\right] \\
& \left.\quad+H_{0}^{p}+H_{1}^{p}+H_{2}^{p}+H_{3}^{p}+H_{4}^{p}+H_{5}^{p}+H_{6}^{p}+H_{7}^{p}+H_{8}^{p}\right) .
\end{aligned}
$$

Proof. Let $H_{i}$ be the polynomials from Theorem 6.11 The formula follows immediately from the fact that $f_{\text {jet }} h_{\text {jet }}=\phi\left(f_{\text {jet }}\right)$.

Next working modulo $p^{2}, h_{\text {jet }}=\left(f_{\text {jet }} h_{\text {jet }}\right) / f_{\text {jet }}$ is $\frac{h_{0}}{f_{0}}+p\left(\frac{-h_{0} f_{1}}{f_{0}^{2}}+\frac{h_{1}}{f_{0}}\right)$, where $f_{0}$ is the coefficient of $p^{0}$ in $f_{\text {jet }}, f_{1}$ is the coefficient of $p, h_{0}$ is the coefficient of $p^{0}$ in $f_{\text {jet }} h_{\text {jet }}$, and $h_{1}$ is the coefficient of $p$. In particular

$$
\begin{aligned}
f_{0}= & {\left[\frac{-72 a_{6}^{p} \delta\left(a_{4}\right)+48 a_{4}^{p} \delta\left(a_{6}\right)}{\Delta^{p}}\right] \gamma_{2 p, p} } \\
& +\frac{1}{\Delta^{p}}\left[-8 a_{4}^{2 p} \mu_{0, p}+72 a_{6}^{p} \mu_{p, p}-48 a_{4}^{p} \mu_{2 p, p}-24 a_{4}^{2 p} \mu_{3 p, 3 p}\right], \\
f_{1}= & \frac{1}{\Delta^{p}}\left[\left((27 \delta(2)+66 \delta(3)) a_{6}^{p} \delta\left(a_{4}\right)\right.\right. \\
& \left.\quad+(-28 \delta(3)-42 \delta(2)) a_{4}^{p} \delta\left(a_{6}\right)\right) \gamma_{2 p, p}+20 \delta(2) a_{4}^{2 p} \mu_{0, p} \\
& \quad+(-27 \delta(2)-66 \delta(3)) a_{6}^{p} \mu_{p, p}+(42 \delta(2)+28 \delta(3)) a_{4}^{p} \mu_{2 p, p} \\
& \left.\quad+18 \delta(2) a_{4}^{p} a_{6}^{p} \mu_{2 p, 3 p}+(8 \delta(3)+24 \delta(2)) a_{4}^{2 p} \mu_{3 p, 3 p}\right] \\
& +\left(H_{0}+H_{1}+H_{2}+H_{3}+H_{4}+H_{5}+H_{6}+H_{7}+H_{8}\right), \\
h_{0}= & \left.\frac{-72 a_{6}^{p^{2}} \delta\left(a_{4}\right)^{p}+48 a_{4}^{p^{2}} \delta\left(a_{6}\right)^{p}}{\Delta^{p^{2}}}\right] \gamma_{2 p, p}\left(a_{4}^{p}, a_{6}^{p}\right) \\
& +\frac{1}{\Delta^{p^{2}}}\left[-8 a_{4}^{2 p^{2}} \mu_{0, p}\left(a_{4}^{p}, a_{6}^{p}\right)+72 a_{6}^{p^{2}} \mu_{p, p}\left(a_{4}^{p}, a_{6}^{p}\right)\right. \\
& \left.-48 a_{4}^{p^{2}} \mu_{2 p, p}\left(a_{4}^{p}, a_{6}^{p}\right)-24 a_{4}^{2 p^{2}} \mu_{3 p, 3 p}\left(a_{4}^{p}, a_{6}^{p}\right)\right], \\
h_{1}= & {\left[\frac{-72 a_{6}^{p^{2}} \delta^{2}\left(a_{4}\right)+48 a_{4}^{p^{2}} \delta^{2}\left(a_{6}\right)}{\Delta^{p^{2}}}\right] \gamma_{2 p, p}\left(a_{4}^{p}, a_{6}^{p}\right)+J_{0} . }
\end{aligned}
$$

Therefore we have the following explicit formulation for $h_{\text {jet }}$ where $J_{0}$ is the polynomial from Theorem 7.3 
Theorem 7.4. The reduction modulo $p^{2}$ of $h_{\text {jet }}$ is

$$
\begin{gathered}
\left(\left(-72 a_{6}^{p^{2}} \delta\left(a_{4}\right)^{p}+48 a_{4}^{p^{2}} \delta\left(a_{6}\right)^{p}\right) \gamma_{2 p, p}\left(a_{4}^{p}, a_{6}^{p}\right)-8 a_{4}^{2 p^{2}} \mu_{0, p}\left(a_{4}^{p}, a_{6}^{p}\right)\right. \\
\left.+72 a_{6}^{p^{2}} \mu_{p, p}\left(a_{4}^{p}, a_{6}^{p}\right)-48 a_{4}^{p^{2}} \mu_{2 p, p}\left(a_{4}^{p}, a_{6}^{p}\right)-24 a_{4}^{2 p^{2}} \mu_{3 p, 3 p}\left(a_{4}^{p}, a_{6}^{p}\right)\right) / \\
\left(\Delta ^ { p ^ { 2 } - p } \left(\left(-72 a_{6}^{p} \delta\left(a_{4}\right)+48 a_{4}^{p} \delta\left(a_{6}\right)\right) \gamma_{2 p, p}\right.\right. \\
\left.\left.-8 a_{4}^{2 p} \mu_{0, p}+72 a_{6}^{p} \mu_{p, p}-48 a_{4}^{p} \mu_{2 p, p}-24 a_{4}^{2 p} \mu_{3 p, 3 p}\right)\right)+p K_{0} \\
+\frac{p\left(-72 a_{6}^{p^{2}} \delta^{2}\left(a_{4}\right)+48 a_{4}^{p^{2}} \delta^{2}\left(a_{6}\right)\right) \gamma_{2 p, p}\left(a_{4}^{p}, a_{6}^{p}\right)}{\Delta^{p^{2}-p}\left(\left(-72 a_{6}^{p} \delta\left(a_{4}\right)+48 a_{4}^{p} \delta\left(a_{6}\right)\right) \gamma_{2 p, p}-8 a_{4}^{2 p} \mu_{0, p}+72 a_{6}^{p} \mu_{p, p}-48 a_{4}^{p} \mu_{2 p, p}-24 a_{4}^{2 p} \mu_{3 p, 3 p}\right)},
\end{gathered}
$$

where $K_{0}$ is

$$
\begin{gathered}
\frac{\Delta^{p} J_{0}}{\left(\left(-72 a_{6}^{p} \delta\left(a_{4}\right)+48 a_{4}^{p} \delta\left(a_{6}\right)\right) \gamma_{2 p, p}-8 a_{4}^{2 p} \mu_{0, p}+72 a_{6}^{p} \mu_{p, p}-48 a_{4}^{p} \mu_{2 p, p}-24 a_{4}^{2 p} \mu_{3 p, 3 p}\right)} \\
-\left(\left(-72 a_{6}^{p^{2}} \delta\left(a_{4}\right)^{p}+48 a_{4}^{p^{2}} \delta\left(a_{6}\right)^{p}\right) \gamma_{2 p, p}\left(a_{4}^{p}, a_{6}^{p}\right)-8 a_{4}^{2 p^{2}} \mu_{0, p}\left(a_{4}^{p}, a_{6}^{p}\right)\right. \\
\left.\quad+72 a_{6}^{p^{2}} \mu_{p, p}\left(a_{4}^{p}, a_{6}^{p}\right)-48 a_{4}^{p^{2}} \mu_{2 p, p}\left(a_{4}^{p}, a_{6}^{p}\right)-24 a_{4}^{2 p^{2}} \mu_{3 p, 3 p}\left(a_{4}^{p}, a_{6}^{p}\right)\right) \\
\times\left(\frac { 1 } { \Delta ^ { p } } \left[\left((27 \delta(2)+66 \delta(3)) a_{6}^{p} \delta\left(a_{4}\right)+(-28 \delta(3)-42 \delta(2)) a_{4}^{p} \delta\left(a_{6}\right)\right) \gamma_{2 p, p}\right.\right. \\
+20 \delta(2) a_{4}^{2 p} \mu_{0, p}+(-27 \delta(2)-66 \delta(3)) a_{6}^{p} \mu_{p, p}+(42 \delta(2)+28 \delta(3)) a_{4}^{p} \mu_{2 p, p} \\
\left.\left.+18 \delta(2) a_{4}^{p} a_{6}^{p} \mu_{2 p, 3 p}+(8 \delta(3)+24 \delta(2)) a_{4}^{2 p} \mu_{3 p, 3 p}\right]+\sum_{i=0}^{8} H_{i}\right) / \\
\left(\left(\left(-72 a_{6}^{p} \delta\left(a_{4}\right)+48 a_{4}^{p} \delta\left(a_{6}\right)\right) \gamma_{2 p, p}\right.\right. \\
\left.\left.-8 a_{4}^{2 p} \mu_{0, p}+72 a_{6}^{p} \mu_{p, p}-48 a_{4}^{p} \mu_{2 p, p}-24 a_{4}^{2 p} \mu_{3 p, 3 p}\right)^{2} \Delta^{p^{2}-2 p}\right) .
\end{gathered}
$$

\section{REFERENCES}

1. M. Barcau and A. Buium, Siegel Differential Modular Forms, Int. Math. Res. Not. (2002), no. 28, 1457-1503. MR1908022 (2003g:11044)

2. A. Buium, Geometry of Fermat Adeles, Preprint, 1999.

3. L Differential Modular Forms, J. Reine Angew. Math. (2000), no. 520, 95-167. MR 1748272 (2002d:11042)

4. _ Arithmetic Differential Invariants, In preparation, 2003.

5. C. Hurlburt, Isogeny Covariant Differential Modular Forms modulo p, Compositio Mathematica 128 (2001), no. 1, 17-34. MR1847663 (2002i:11053)

6. J. Silverman, The Arithmetic of Elliptic Curves, Graduate Texts in Mathematics, vol. 106, Springer Verlag, 1986. MR0817210 (87g:11070)

Department of Mathematics, Northern Illinois University, DeKalb, Illinois 60115

E-mail address: hurlburt@math.niu.edu 